\title{
Convergence of Finite Volume Approximations for a Nonlinear Elliptic-Parabolic Problem: a "Continuous" Approach
}

\author{
Boris Andreianov ${ }^{1}$, Michaël Gutnic ${ }^{2}$ and Petra Wittbold ${ }^{2}$
}

December 21, 2003

\begin{abstract}
We study the approximation by finite volume methods of the model parabolic-elliptic problem $b(v)_{t}=$ $\operatorname{div}\left(|D v|^{p-2} D v\right)$ on $(0, T) \times \Omega \subset \mathbb{R} \times \mathbb{R}^{d}$ with an initial condition and the homogeneous Dirichlet boundary condition. Because of the nonlinearity in the elliptic term, a careful choice of the gradient approximation is needed.

We prove the convergence of discrete solutions to a solution of the continuous problem as the discretization step $h$ tends to 0 , under the main hypotheses that the approximation of the operator $\operatorname{div}\left(|D v|^{p-2} D v\right)$ provided by the finite volume scheme is still monotone and coercive, and that the gradient approximation is exact on the affine functions of $x \in \Omega$. An example of such a scheme is given for a class of two-dimensional meshes dual to triangular meshes, in particular for structured rectangular and hexagonal meshes.

The proof uses the rewriting of the discrete problem under a "continuous" form. This permits us to directly apply the Alt-Luckhaus variational techniques known in the continuous case.
\end{abstract}

Key words: doubly nonlinear elliptic-parabolic equations; finite volume methods; convergence of approximate solutions; continuous approach.

AMS subject classification: 35J60, 35K55, 35K65, 35M10, 65M12, 76M12.

\footnotetext{
${ }^{1}$ LATP, CMI, Université de Provence, Technopole de Château-Gombert, 39, rue Frédéric Joliot-Curie, 13453 Marseille Cedex 13, France. E-mail: borisa @math.univ-fcomte.fr

${ }^{2}$ IRMA, Université Louis Pasteur, 7 rue René Descartes, 67084 Strasbourg Cedex, France.

E-mails: gutnic,wittbold@math.u-strasbg.fr
} 


\section{Contents}

1 Introduction 3

2 THE NUMERICAL METHOD 4

2.1 Finite volume meshes, Discrete gradients AND Finite VolUME SCHEMES FOR PROBLEM $(1.1) \ldots \ldots \ldots \ldots \ldots$

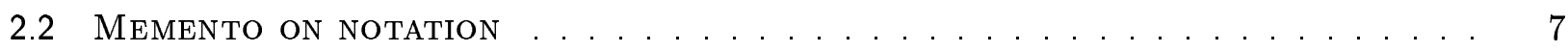

2.3 AdMISSIBLE FLUX APPROXIMATIONS $\ldots \ldots \ldots \ldots \ldots \ldots \ldots \ldots$

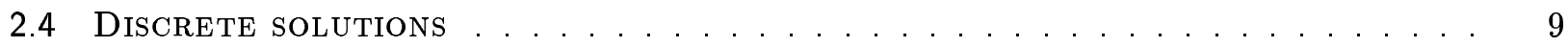

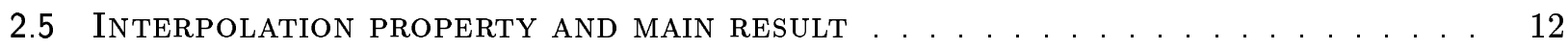

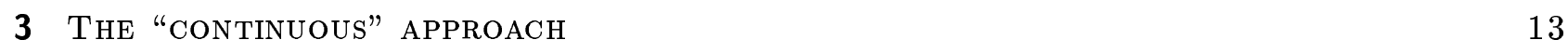

3.1 Interpolated gradient and the "COntinuous" form of the SCheme . . . . 13

3.2 Properties of the interPolated GRAdient AND CONSISTENCY $\ldots \ldots \ldots$

4 Proof of Theorem $2 \quad 19$

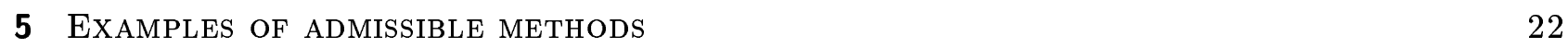

5.1 ON DISCRETE GRADIENTS . . . . . . . . . . . . . . . . . . . . . . . . 22

5.2 ON INTERPOLATED SOLUTIONS . . . . . . . . . . . . . . . . . . . . 24

6 Generalisation to a case with convection 27

A Appendix: PROOFs of AUXILIARY RESUlts 29 


\section{INTRODUCTION}

Let $\Omega$ be an open bounded polygonal domain in $\mathbb{R}^{d}, d \geq 1$ and $T>0$. We consider the initial boundary value-problem for a system of nonlinear elliptic-parabolic equations:

$$
\begin{cases}b(v)_{t}=\operatorname{div} a_{p}(D v) & \text { on } Q=(0, T) \times \Omega, \\ v=0 & \text { on } \Sigma=(0, T) \times \partial \Omega, \\ b(v)(0, \cdot)=u^{0} & \text { on } \Omega\end{cases}
$$

where $1<p<\infty$ and $\operatorname{div} a_{p}(D v)=\operatorname{div}\left(|D v|^{p-2} D v\right)$ is the $N$-dimensional $p$-Laplacian, $N \geq 1$, i.e.,

We assume that

$$
a_{p}: \xi=\left(\xi_{1}, \ldots, \xi_{N}\right) \in\left(\mathbb{R}^{d}\right)^{N} \mapsto|\xi|^{p-2} \xi=\left(\sum_{i, j}\left|\xi_{i}^{j}\right|^{2}\right)^{p / 2-1}\left(\xi_{1}, \ldots, \xi_{N}\right) \in\left(\mathbb{R}^{d}\right)^{N}
$$

$$
\left\{\begin{array}{l}
b: \mathbb{R}^{N} \rightarrow \mathbb{R}^{N} \text { is continuous cyclically monotone, i.e., } \\
\text { there exists a convex differentiable function } \Phi: \mathbb{R}^{N} \rightarrow \mathbb{R} \text { such that } b=\nabla \Phi,
\end{array}\right.
$$

normalized by $b(0)=0$ and $\Phi(0)=0$. Moreover, we assume

$$
u^{0} \in L^{1}(\Omega)^{N} \quad \text { with } \quad \Psi\left(u_{0}\right) \in L^{1}(\Omega),
$$

where $\Psi$ is the Legendre transform of $\Phi$ given by

$$
\Psi: z \in \mathbb{R}^{N} \mapsto \sup _{\sigma \in \mathbb{R}^{N}} \int_{0}^{1}(z-b(s \sigma)) \sigma d s=\sup _{\sigma \in \mathbb{R}^{N}}(\sigma z-\Phi(\sigma)) .
$$

Equations of elliptic-parabolic type (1.1) arise as models of flow of fluids through porous media (cf., e.g., $[5,12])$. They have already been studied extensively in the literature in the last decade from a theoretical point of view (cf., e.g., [1, 21, 22, 12, 6, 26, 7, 10, 2]). Existence of weak solutions of general systems of elliptic-parabolic equations has been proved in [1], using Galerkin approximations and time-discretization. Similar results have been obtained later by other authors using different methods (e.g., using a semigroup approach as in $[6,7]$ in the case $N=1$ ).

In particular, it is known that in the case of the system (1.1), for any $u_{0}$ satisfying (1.3), there exists a weak solution of (1.1), where the weak solution is defined as follows. Denote by $E$ the Banach space $L^{p}\left(0, T ; W_{0}^{1, p}(\Omega)\right)^{N}$ and by $E^{\prime}$ its dual; $E^{\prime}=L^{p^{\prime}}\left(0, T ; W^{-1, p^{\prime}}(\Omega)\right)^{N}$ where $p^{\prime}=p /(p-1)$ is the conjugate exponent of $p$. Denote by $\langle\cdot, \cdot\rangle_{E^{\prime}, E}$ the duality pairing between $E^{\prime}$ and $E$.

Definition 1 (Weak solution) A function $v \in E$ is a weak solution of Problem (1.1), if $b(v) \in L^{\infty}\left(0, T ; L^{1}(\Omega)\right)^{N}$, and $b(v)_{t} \in \mathcal{D}^{\prime}(Q)^{N}$ can be extended to a functional $\chi$ on $E$ satisfying

$$
\begin{array}{cl}
<\chi, \phi>_{E^{\prime}, E}+\iint_{Q} a_{p}(D v) \cdot D \phi=0 \quad \text { for all } \phi \in E \\
<\chi, \xi>_{E^{\prime}, E}=-\iint_{Q} b(v) \xi_{t}-\int_{\Omega} u_{0}(\cdot) \xi(0, \cdot) & \text { for all } \xi \in E \text { with } \\
\xi_{t} \in L^{\infty}(Q)^{N}, \xi(T, \cdot)=0 .
\end{array}
$$

Note that if $v$ is a weak solution of (1.1), then, by the "chain rule" lemma of [1], one has

$$
\begin{aligned}
& B(v) \in L^{\infty}\left(0, T ; L^{1}(\Omega)\right)^{N}, \quad \text { where } \\
& B: z \in \mathbb{R}^{N} \mapsto b(z) z-\Phi(z) \equiv \int_{0}^{1}(b(z)-b(s z)) z d s \equiv \Psi(b(z)) \in \mathbb{R} .
\end{aligned}
$$


From the results of $[26,10]$ it also follows that, in the scalar case $N=1$, there is uniqueness of a weak solution of (1.1). To our knowledge, the question of uniqueness is open in the case $N \geq 2$.

In this paper we study the convergence of time implicit approximations by finite volume numerical schemes for the model nonlinear elliptic-parabolic problem (1.1). Finite volume methods are well suited for numerical simulation of processes where extensive quantities are conserved, and they are popular methods among engineers in hydrology where equations of this type arise. Therefore justification of convergence of this numerical approximation process is of particular interest. In [17] the finite volume method has been studied and convergence of this approximation procedure has been proved for Problem (1.1) in the particular case $p=2, N=1$. The same method has also been studied for this equation (i.e., $p=2, N=1$ ) in the presence of an additional convection term (cf. [18, 14]), and for a nonlinear diffusion problem in [16]. To our knowledge, in the case $p \neq 2$, only the convergence of finite element methods has been studied (cf. $[19,11,4,20]$ and their references).

Let us emphasize that our main object is not only to prove the convergence of some finite volume methods for (1.1), but also to develop a "continuous" approach for this proof. The main idea of this adaptation is to rewrite the discrete finite volume scheme under an equivalent continuous form and to apply known stability techniques for the continuous equation (cf. [1] and [2, Chap.V] for the version we use) in order to get convergence of the discrete solutions to a solution of the continuous problem. The "continuous" approach and the convergence result have already been presented in [3].

In Section 2, we describe the finite volume schemes and in particular the admissible flux approximations we use. We show the existence and uniqueness of the solution of a finite volume scheme and give some a priori estimates on discrete solutions. Then we state the convergence result. In Section 3, we show in Proposition 2 that the solution of a finite volume scheme, originally satisfying a discrete system of algebraic equations, also verifies a "continuous" formulation similar to (1.4),(1.5). This representation makes clear in which sense finite volume schemes approximate the elliptic operator in (1.1); we prove that this approximation is consistent. In Section 4 we prove the convergence theorem, passing to the limit in the "continuous" formulation of Proposition 2. In Section 5, we analyze the two admissibility conditions imposed in Section 2. For $d=2$, we propose a scheme on meshes dual to triangular meshes which enter into our framework; in particular, we have the convergence result on structured rectangular and hexagonal meshes.

We consider the $p$-Laplacian as a prototype of a class of the so-called Leray-Lions-type operators; in Section 6 , we discuss the extension of the techniques presented above to a particular case of the $p$-Laplacian operator with convection, studied in [12].

The Appendix A contains the proofs of the auxiliary results used in Section 4. In order to simplify the notation, we restrict the exposition to the scalar equation case $(N=1)$.

\section{The NUMERICAL METHOD}

In order to construct approximate solutions to Problem (1.1), we will use the implicit discretization in time and a finite volume scheme in space. 


\subsection{Finite volume meshes, Discrete Gradients AND Finite VOLUME SCHEMES FOR PROBLEM (1.1)}

Let $\Omega$ be an open bounded polygonal subset of $\mathbb{R}^{d}$. A finite volume mesh $\mathcal{T}$ of $\Omega$ is given by: a family of open polygonal convex subsets of $\Omega$ with positive measure, called "control volumes"; a family of subsets of $\bar{\Omega}$ contained in hyperplanes of $\mathbb{R}^{d}$, with positive $(d-1)$-measure (these are the interfaces between control volumes); and a family of points of $\bar{\Omega}$, one per control volume (these are the "centers" of the volumes). For a volume $K$ with center $x_{K} \in \bar{K}$, the interfaces contained in $\partial \Omega$ are considered as additional "boundary" volumes, unless $x_{K} \in \partial \Omega$.

For the sake of simplicity, we shall denote by $\mathcal{T}$ the family $(K)_{K \in \mathcal{T}}$ of control volumes; $\left(x_{K}\right)_{K \in \mathcal{T}}$ denotes the family of their centers. The set of all volumes $K$ such that $x_{K} \in \partial \Omega$ is denoted by $\mathcal{T}_{\text {ext }}$, and the set of all volumes $K$ with $x_{K} \in \Omega$ is denoted by $\mathcal{T}_{\text {int }}$. The set of interfaces $K L$ such that $K$ or $L$ or both belong to $\mathcal{T}_{\text {int }}$ is denoted by $\mathcal{E}$, and $K L$ denotes the interface between two neighbours $K, L \in \mathcal{T}$. For all $K L, \widehat{K L}$ denotes the "diamond" over $K L$, i.e., the smallest convex set of $\mathbb{R}^{d}$ containing $K L, x_{K}$ and $x_{L}$. Whenever we use $K, K L$ or $n$ to index objects and make summations, we mean that $K \in \mathcal{T}$, $K L \in \mathcal{E}$ and $n \in\{1, \ldots,[T / k]+1\}$, where $k$ is the time step of the scheme.

Following [15], we give the following definition.

Definition 2 (Finite volume mesh) We say that $\mathcal{T}$ is a finite volume mesh of $\Omega$ if the following hold:

(2i) The closure of the union of all control volumes is $\bar{\Omega}$.

(2ii) For any $(K, L) \in(\mathcal{T})^{2}$ with $K \neq L$, either the $(d-1)$-dimensional measure of $\bar{K} \cap \bar{L}$ is 0 or $\bar{K} \cap \bar{L}=\bar{\sigma}$ for some $\sigma \in \mathcal{E}$ (in which case we denote $\sigma=K L=L \mid K$ ).

(2 iii) For any $K \in \mathcal{T}$, there exists a subset $\mathcal{E}_{K}$ of $\mathcal{E}$ such that $\partial K=\cup_{\sigma \in \mathcal{E}_{K}} \bar{\sigma}$. Furthermore, $\mathcal{E}=\cup_{K \in \mathcal{T}} \mathcal{E}_{K}$. We will denote by $\mathcal{N}_{K}$ the set of volumes adjacent to $K$; i.e., $\mathcal{N}_{K}=\left\{L \in \mathcal{T}, K L \in \mathcal{E}_{K}\right\}$.

(2iv) The family of points $\left(x_{K}\right)_{K}$ is such that $x_{K} \in \bar{K}$ for all $K \in \mathcal{T}$, and it is assumed that the straight line joining $x_{K}$ and $x_{L}$ is orthogonal to $K L$ whenever $L \in \mathcal{N}_{K}$.

Denote by $\mathfrak{m}(K)$ and $\mathfrak{d}(K)$ the $d$-dimensional measure and the diameter of $K \in \mathcal{T}$, respectively; and denote by $m(K L)$, the $(d-1)$-dimensional measure of $K L \in \mathcal{E}$. A mesh $\mathcal{T}$ is characterized, in particular, by the following numbers:

$$
\begin{array}{ll}
\operatorname{size}(\mathcal{T})=\max _{K} \mathfrak{d}(K), & \zeta^{*}(\mathcal{T})=\min _{K} \min _{\sigma \in \mathcal{E}_{K}} \frac{\operatorname{dist}\left(x_{K}, \sigma\right)}{\mathfrak{d}(K)}, \\
M(\mathcal{T})=\max _{K} \operatorname{card}\left(\mathcal{E}_{K}\right), & \zeta_{*}(\mathcal{T})=\frac{\min _{K} \min _{\sigma \in \mathcal{E}_{K}} \operatorname{dist}\left(x_{K}, \sigma\right)}{\operatorname{size}(\mathcal{T})} .
\end{array}
$$

A finite volume method for (1.1) requires a family $\left(\left(\mathcal{T}^{h}, k^{h}\right)\right)_{h}$ of meshes and corresponding time steps $k^{h}>0$ such that both the size of the mesh and the time step go to zero. We will assume in our notation that the family is parametrized with $h$ in some subset of $(0,1)$ whose closure contains zero, and $\operatorname{size}\left(\mathcal{T}^{h}\right)+k^{h} \leq h$. A couple $\left(\mathcal{T}^{h}, k^{h}\right)$ will be called a space-time grid.

In relation to a family $\left(\left(\mathcal{T}^{h}, k^{h}\right)\right)_{h}$, we define the numbers

$$
M=\sup _{h} M\left(\mathcal{T}^{h}\right) \in \overline{\mathbb{N}}, \quad \zeta^{*}=\inf _{h} \zeta^{*}\left(\mathcal{T}^{h}\right) \in \mathbb{R}^{+}, \quad \text { and } \quad \zeta_{*}=\inf _{h} \zeta_{*}\left(\mathcal{T}^{h}\right) \in \mathbb{R}^{+} .
$$




\section{Definition 3 (Proportionality of meshes)}

We say that the family of meshes $\left(\mathcal{T}^{h}\right)_{h}$ is weakly proportional if $M<\infty$ and $\zeta^{*}>0$. We say that the family of meshes $\left(\mathcal{T}^{h}\right)_{h}$ is strongly proportional if, in addition, $\zeta_{*}>0$.

Weak proportionality is standard (cf. [18]). Strong proportionality is a technical assumption which ensures that $\left(\mathcal{T}^{h}\right)_{h}$ has the interpolation property (cf. Sections 2.5 and 5.2).

Given a grid $\left(\mathcal{T}^{h}, k^{h}\right)$, to each time-space volume $Q_{K}^{n}=I^{n} \times K, I^{n}=(k(n-1), k n)$ one associates an unknown value $v_{K}^{n} \in \mathbb{R}^{N}$. In order to obtain a finite volume scheme for (1.1), one "integrates" the equation in (1.1) over each grid volume $Q_{K}^{n}$. The time derivative in the left-hand side is approximated by the corresponding finite difference. On the right-hand side, one uses the Green formula and then needs to replace the flux on the lateral boundary of $Q_{K}^{n}$ by some function of the unknowns $\left(v_{K}^{n}\right)_{K, n}$. For Problem (1.1), this amounts to finding a substitution for $D v$ in the expression $\int_{I} n_{\times K L} a_{p}(D v) \cdot \nu_{K, L}$ (where $\nu_{K, L}$ is the unit normal vector to $K L$ pointing from $K$ into $L$ ). We will assume that this substitute is in $L^{p}$ on each interface $I^{n} \times K L$, typically constant in time and piecewise constant in space. We therefore consider "discrete gradient" operators $\mathcal{D}^{h}$ of the form

$$
\left\{\begin{array}{l}
\mathcal{D}^{h}:\left(v_{K}^{n}\right)_{K, n} \mapsto\left(D_{K L}^{n}\right)_{K L, n} \\
D_{K L}^{n} \in L^{p}\left(I^{n} \times K L\right) \quad \text { for all } K L, n
\end{array}\right.
$$

It seems natural, though not necessary, to require that $\mathcal{D}^{h}$ be a linear operator.

A finite volume scheme for (1.1) is defined by a grid $\left(\mathcal{T}^{h}, k^{h}\right)$ and a discrete gradient $\mathcal{D}^{h}$ associated with the grid. Finally, a finite volume method for $(1.1)$ is given by a family $\left(\left(\mathcal{T}^{h}, k^{h}, \mathcal{D}^{h}\right)\right)_{h}$ of grids and associated discrete gradient operators $\mathcal{D}^{h}$. In Sections 2.3 and 2.5 we state the admissibility conditions for such methods.

Now we are able to write the equations for a scheme $\left(\mathcal{T}^{h}, k^{h}, \mathcal{D}^{h}\right)$ :

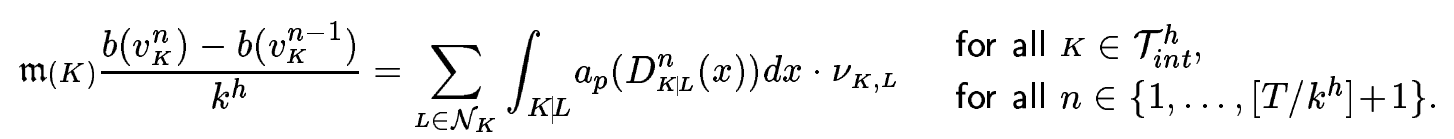

The homogeneous Dirichlet boundary condition is taken into account by assigning

$$
v_{K}^{n}=0 \quad \text { for all } K \in \mathcal{T}_{\text {ext }}^{h}, \quad \text { for all } n \in\left\{1, \ldots,\left[T / k^{h}\right]+1\right\} .
$$

The initial condition is given by any values $v_{K}^{0} \in b^{-1}\left(u_{K}^{0}\right)$, where

$$
u_{K}^{0}=\frac{1}{\mathfrak{m}(K)} \int_{K} u^{0} \quad \text { for all } K \in \mathcal{T}_{\text {int }}^{h} .
$$

We denote by $u_{0}^{h}$ the piecewise constant initial function $\sum_{K} u_{K}^{0} \mathbb{1}_{K}$, where $\mathbb{1}_{K}$ is the characteristic function of the set $K$. Other choices of $u_{K}^{0}$ are possible, provided one has $u_{0}^{h} \rightarrow u_{0}$ a.e. on $\Omega$ and $\Psi\left(u_{0}^{h}\right) \rightarrow \Psi\left(u_{0}\right)$ in $L^{1}(\Omega)$ as $h \rightarrow 0$, where $\Psi$ is defined in the introduction. These properties hold for $u_{K}^{0}$ given by (2.11), due to the convexity of $\Psi$.

We denote by $\left(\mathcal{S}^{h}\right)$ the system (2.9),(2.10),(2.11) corresponding to a given finite volume scheme $\left(\mathcal{T}^{h}, k^{h}, \mathcal{D}^{h}\right)$. 


\subsection{MEMENTO ON NOTATiON}

In this section we collect the most used notation related to the finite volume schemes.

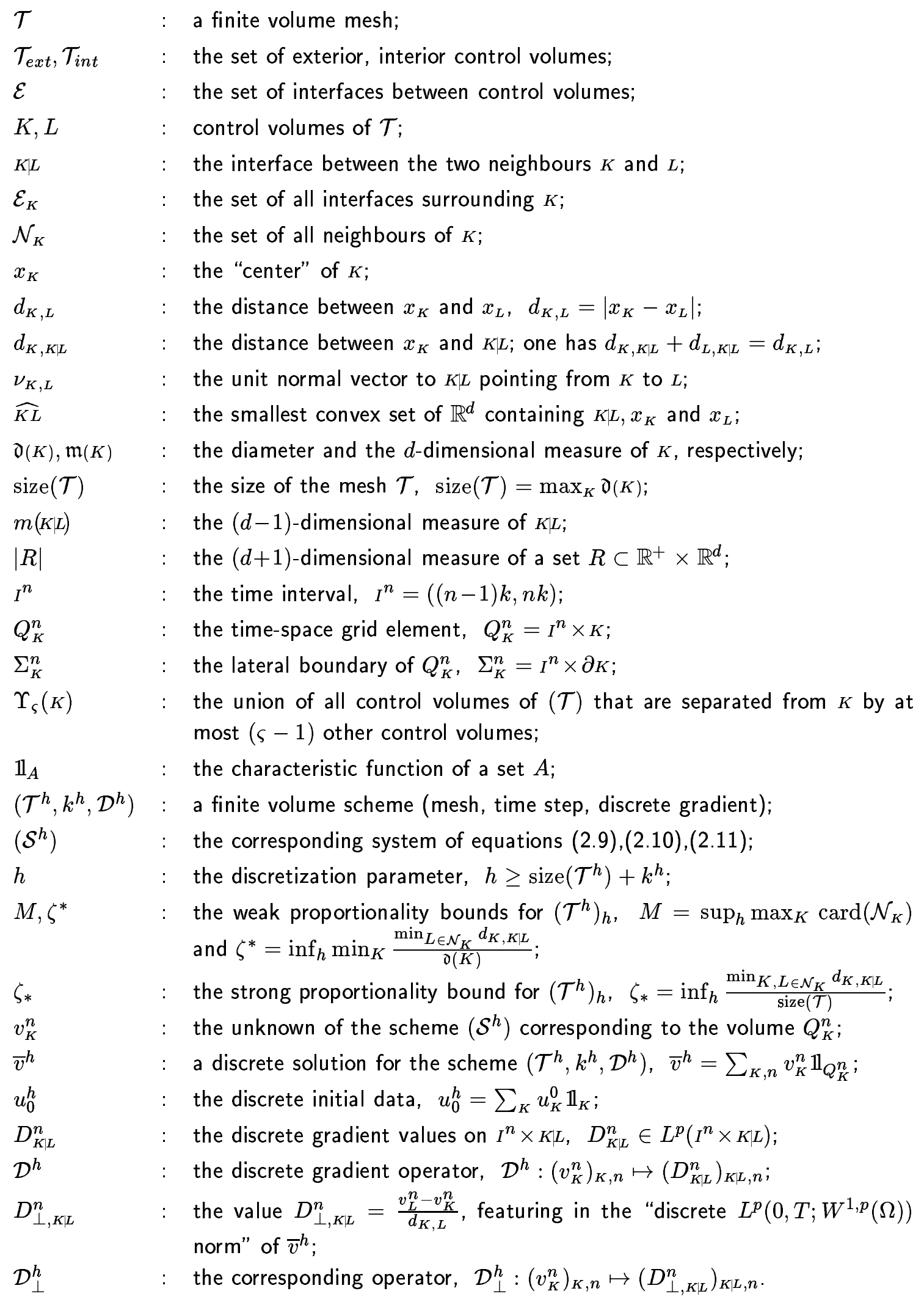


It is convenient to extend $\mathcal{D}^{h}$ (as well as $\mathcal{D}_{\perp}^{h}$ ) to an operator acting from $E$ into $L^{p}\left(Q\right.$ ). Let $\mathcal{P}^{h}$ be the operator from $\Omega$ to $\bigcup K L$ which projects $x \in K$ on $\partial_{K}$ along the ray joining $x_{K}$ to $x$. The lifting operator $\mathcal{L}^{h}$ is then defined by We define the appropriate lifting operator $\mathcal{L}^{h}$ and averaging operator $\mathcal{M}^{h}$ by

$$
\begin{aligned}
& \mathcal{L}^{h}\left[\left(D_{K \mid L}^{n}\right)_{K \mid L, n}\right](t, x)=\sum_{K \mid L, n} D_{K \mid L}^{n}\left(\mathcal{P}^{h}(x)\right) \mathbb{1}_{I^{n_{\times} \widehat{K L}}}(t, x), \\
& \mathcal{M}^{h}: \eta \in L^{1}(Q) \mapsto \mathcal{M}^{h}[\eta]=\left(\eta_{K}^{n}\right)_{K, n} \subset \mathbb{R}^{N}, \quad \eta_{K}^{n}=\frac{1}{\left|Q_{K}^{n}\right|} \iint_{Q_{K}^{n}} \eta .
\end{aligned}
$$

We will abusively write $\mathcal{D}^{h}$ for the operators $\mathcal{D}^{h}, \mathcal{L}^{h} \circ \mathcal{D}^{h}$, and $\mathcal{L}^{h} \circ \mathcal{D}^{h} \circ \mathcal{M}^{h}$; and the same for $\mathcal{D}_{\perp}^{h}$.

The following notations, specific to the "continuous" approach, are introduced in Sections 2.5 and 3.1.

$u^{h} \quad: \quad$ the continuous in $t$ interpolation of $b\left(\bar{v}^{h}\right)$, affine on each time interval $I^{n}$;

$v^{h} \quad$ : an interpolated solution in $E$ for $\bar{v}^{h}$ (cf. Definition 5);

$\mathcal{G}^{h} \quad$ : the interpolated gradient operator produced by the scheme $\left(\mathcal{T}^{h}, k^{h}, \mathcal{D}^{h}\right)$ (cf. Definition 6);

$\mathcal{A} \quad$ : the elliptic operator in (1.1), $\mathcal{A}: \eta \in E \mapsto-\operatorname{div} a_{p}(D \eta) \in E^{\prime} ;$

$\mathcal{A}^{h}$ : the finite volume approximation of $\mathcal{A}$ produced by the scheme $\left(\mathcal{T}^{h}, k^{h}, \mathcal{D}^{h}\right)$, given by $\mathcal{A}^{h}: \eta \in E \mapsto-\operatorname{div} a_{p}\left(\mathcal{G}^{h}[\eta]\right) \in E^{\prime}$.

\subsection{Admissible flux approximations}

For simplicity, we consider only the gradients that yield fully implicit schemes; in this case $\mathcal{D}^{h}, \mathcal{D}_{\perp}^{h}$ act independently on each set $\left(v_{K}^{n}\right)_{K}$, and the dependence on $n$ does not matter for their definition.

Let us introduce the operator $\mathcal{D}_{\perp}^{h}$, which appears naturally in the a priori estimates of Section 2.4:

$$
\mathcal{D}_{\perp}^{h}:\left(v_{K}\right)_{K} \mapsto\left(D_{\perp, K \mid L}\right)_{K L}, \quad D_{\perp, K \mid L}=\frac{v_{L}-v_{K}}{d_{K, L}} \in \mathbb{R} .
$$

For $\varsigma \in \mathbb{N}$, denote by $\Upsilon_{\varsigma}(K)$ the union of all control volumes of $\mathcal{T}$ that are separated from $K$ by at most $(\varsigma-1)$ other control volumes; for instance, $\Upsilon_{1}(K)=\bigcup_{L \in \mathcal{N}_{K}} L$. The choice of $\varsigma$ corresponds to the choice of control volumes that are really involved in the construction of $\mathcal{D}^{h}$ on $\partial_{K}$.

Now we can make precise the assumptions on discrete gradient operators of the form (2.8).

Definition 4 (Admissible gradient approximation) Let $\left(\mathcal{T}^{h}, \mathcal{D}^{h}\right)_{h}$ be a family of finite volume meshes and corresponding discrete gradient operators. The gradient approximation provided by $\mathcal{D}^{h}$ is admissible if the following hold.

(4i) $\mathcal{D}^{h}$ is linear and injective;

(4ii) $\mathcal{D}^{h}$ provides a strictly monotone scheme; i.e., for all $\left(v_{K}\right)_{K},\left(\widetilde{v}_{K}\right)_{K} \subset\left(\mathbb{R}^{d}\right)^{N}$ that do not coincide,

$$
\frac{1}{d} \sum_{K L}\left(\left(v_{L}-v_{K}\right)-\left(\widetilde{v}_{L}-\widetilde{v}_{K}\right)\right) \int_{K L}\left(a_{p}\left(D_{K L}(x)\right)-a_{p}\left(\widetilde{D}_{K \mid L}(x)\right)\right) d x \cdot \nu_{K, L}>0
$$

where $\left(D_{K \mid L}\right)_{K \mid L}=\mathcal{D}^{h}\left[\left(v_{K}\right)_{K}\right],\left(\widetilde{D}_{K \mid L}\right)_{K L L}=\mathcal{D}^{h}\left[\left(\widetilde{v}_{K}\right)_{K}\right]$;

(4 iii) $\mathcal{D}^{h}$ provides a scheme coercive at zero; i.e., there exists a constant $C_{*}>0$, independent of $h$, such that for all $\left(v_{K}\right)_{K} \subset\left(\mathbb{R}^{d}\right)^{N}$ and $\left(D_{K L}\right)_{K L}=\mathcal{D}^{h}\left[\left(v_{K}\right)_{K}\right]$, one has

$$
\frac{1}{d} \sum_{K L}\left(v_{L}-v_{K}\right) \int_{K L L} a_{p}\left(D_{K \mid L}(x)\right) d x \cdot \nu_{K, L} \geq C_{*}\left\|\mathcal{D}_{\perp}^{h}\left[\left(v_{K}\right)_{K}\right]\right\|_{L^{p}(\Omega)}^{p} ;
$$


and there exists $\varsigma \in \mathbb{N}$, independent of $h$, such that the following hold.

(4iv) For each $h, \mathcal{D}^{h}$ is consistent with affine functions. More exactly, assume that, for $k \in \mathcal{T}^{h}$ given, there exists an affine function $w$ on $\Omega$ such that $v_{L}=\frac{1}{\mathfrak{m}(L)} \int_{L} w$ whenever $L \subset \Upsilon_{\varsigma}(K)$. Then $D_{K L}(x)=D w=$ const for all $x \in K L$ for all $L \in \mathcal{N}_{K}$.

(4v) There exists a constant $C^{*}$, independent of $h$, such that, for all $\widetilde{K} \in \mathcal{T}^{h}$ and all sets of values $\left(v_{K}\right)_{K}$ of $\mathbb{R}^{N}, \int_{\widetilde{K}}\left|\mathcal{D}^{h}\left[\left(v_{K}\right)_{K}\right]\right|^{p} \leq C^{*} \int_{\Upsilon_{\varsigma}(\widetilde{K})}\left|\mathcal{D}_{\perp}^{h}\left[\left(v_{K}\right)_{K}\right]\right|^{p}$.

Conditions (4ii) and (4iv) imply strong restrictions on the gradient approximation. We provide some examples of methods with admissible gradient approximation in Section 5.1.

\subsection{DisCRETE SOLUTIONS}

Recall that we consider as unknowns the values $v_{K}^{n}$ on $K \in \mathcal{T}_{\text {int }}$, assigning $v_{K}^{n}$ to be zero in $K \in \mathcal{T}_{\text {ext }}$. We will repeatedly use the following "summation by parts" formula (cf., e.g., [15]).

Remark 1 (Summation by parts) Let $\mathcal{T}$ be a finite volume mesh of $\Omega$ in the sense of Definition 2. Let $\left(v_{K}\right)_{K \in \mathcal{T}} \subset \mathbb{R}^{N},\left(F_{K, L}\right)_{(K, L) \in \mathcal{T}^{2}} \subset \mathbb{R}^{N}$. Assume $v_{K}=0$ for all $K \in \mathcal{T}_{\text {ext }}$ and $F_{K, L}=-F_{L, K}$ for all $K L \in \mathcal{E}$. Then

$$
\sum_{K} v_{K} \sum_{L \in \mathcal{N}_{K}} F_{K, L}=\sum_{K \mid L}\left(v_{K}-v_{L}\right) F_{K, L} .
$$

If $\left(v_{K}^{n}\right)_{K, n}$ verifies $\left(\mathcal{S}^{h}\right)$, we say that the function $\bar{v}^{h}=\sum_{K, n} v_{K}^{n} \mathbb{1}_{Q_{K}^{n}}$ is the corresponding discrete solution. We prove the discrete version of the $L^{p}\left(0, T ; W_{0}^{1, p}(\Omega)\right.$ )-a priori estimate on $\bar{v}^{h}$ (which is exactly the estimate on $\mathcal{D}_{\perp}^{h}\left[\bar{v}^{h}\right]$ in $L^{p}$ ) and the discrete version of (1.6).

Proposition 1 (A priori estimates) Let $\left(\left(\mathcal{T}^{h}, k^{h}\right)\right)_{h}$ be a family of finite volume grids. Let $\left(\mathcal{D}^{h}\right)_{h}$ be a family of corresponding discrete gradient operators satisfying Property (4iii) of Definition 4. Then for any solution $\bar{v}^{h}$ of the discrete problem $\left(\mathcal{S}^{h}\right)$, there exists a constant $C$ which depends only on $p, d, \Omega, T$, on $C_{*}$ in (4iii), and on $\left\|\Psi\left(u_{0}\right)\right\|_{L^{1}(\Omega)}$ such that

(i) $\left\|\mathcal{D}_{\perp}^{h}\left[\bar{v}^{h}\right]\right\|_{L^{p}(Q)}^{p}=\frac{1}{d} \sum_{K L, n} m(K L) d_{K, L}\left|\frac{v_{L}^{n}-v_{K}^{n}}{d_{K, L}}\right|^{p} \leq C$;

(ii) $\left\|B\left(\bar{v}^{h}\right)\right\|_{L^{\infty}\left(0, T ; L^{1}(\Omega)\right)}=\sup _{n \in\left\{1, \ldots,\left[T / k^{h}\right]+1\right\}} \sum_{K} \mathfrak{m}(K) B\left(v_{K}^{n}\right) \leq C$.

Proof : Take $i \in\left\{1, \ldots,\left[T / k^{h}\right]+1\right\}$ and multiply each term in (2.9) by $v_{K}^{i}$. By (4iii), using Remark 1 and (2.10), one gets

$$
\sum_{K} \mathfrak{m}(K)\left(b\left(v_{K}^{i}\right)-b\left(v_{K}^{i-1}\right)\right) v_{K}^{i}+C_{*} k^{h} d \int_{\Omega}\left|\mathcal{D}_{\perp}^{h}\left[\bar{v}^{h}\right]\right|^{p} \leq 0 .
$$

By the convexity of $\Phi$, one has $\left(b\left(v_{K}^{i}\right)-b\left(v_{K}^{i-1}\right)\right) v_{K}^{i} \geq B\left(v_{K}^{i}\right)-B\left(v_{K}^{i-1}\right)$. Summing over $i$ from 1 to $n \in\left\{1, \ldots,\left[T / k^{h}\right]+1\right\}$ and taking into account the convexity of $\Psi$, we infer

$$
\begin{aligned}
\sum_{K} \mathfrak{m}(K) & B\left(v_{K}^{n}\right)+C_{*} d \int_{0}^{n k^{h}} \int_{\Omega}\left|\mathcal{D}_{\perp}^{h}\left[\bar{v}^{h}\right]\right|^{p} \leq \\
\leq & \sum_{K} \mathfrak{m}(K) \Psi\left(u_{K}^{0}\right)=\sum_{K} \mathfrak{m}(K) \Psi\left(\frac{1}{\mathfrak{m}(K)} \int_{K} u^{0}\right) \leq \int_{\Omega} \Psi\left(u^{0}\right) .
\end{aligned}
$$


Next, let us prove the discrete version of the Poincare inequality and of the compact embedding of $W^{1, p}(\Omega)$ in $L^{1}(\Omega)$ and in $L^{p}(\Omega)$. Note that in (i) and (ii), we do not need any proportionality assumptions on the mesh.

Lemma 1 (Discrete Poincaré inequality) Let $\Omega \subset \mathbb{R}^{d}$ be a polygonal domain of diameter $\mathfrak{d}(\Omega)$, and let $\mathcal{T}$ be a finite volume mesh of $\Omega$. Let $\bar{v}^{h}=\sum_{K} v_{K} \mathbb{1}_{K}$ such that $\left(v_{K}\right)_{K \in \mathcal{T}} \subset \mathbb{R}$ and $v_{K}=0$ for all $K \in \mathcal{T}_{\text {ext }}$. Then there exists a constant $C$ which depends only on $p$ and $d$ such that

(i) $\left\|\bar{v}^{h}\right\|_{L^{p}(\Omega)} \leq C \mathfrak{d}(\Omega)\left\|\mathcal{D}_{\perp}^{h}\left[\bar{v}^{h}\right]\right\|_{L^{p}(\Omega)} ;$

(ii) for all $\Delta>0, \quad \sup _{|\Delta x| \leq \Delta} \int_{\mathbb{R}^{d}}\left|\bar{v}^{h}(x+\Delta x)-\bar{v}^{h}(x)\right| d x \leq \Delta \times\left\|\mathcal{D}_{\perp}^{h}\left[\bar{v}^{h}\right]\right\|_{L^{1}(\Omega)}$.

(iii) there exists a constant $\widetilde{C}$ depending on $p, d$ and on $\zeta_{*}(\mathcal{T})$ such that for all $\Delta>0, \quad \sup _{|\Delta x| \leq \Delta} \int_{\mathbb{R}^{d}}\left|\bar{v}^{h}(x+\Delta x)-\bar{v}^{h}(x)\right|^{p} d x \leq \Delta(\Delta+2 \operatorname{size}(\mathcal{T}))^{p-1} \times \widetilde{C}\left\|\mathcal{D}_{\perp}^{h}\left[\bar{v}^{h}\right]\right\|_{L^{p}(\Omega)}^{p}$.

Proof: (i) For $x \in \Omega$, set $\psi_{K L}(x)=1$, in the case that the orthogonal projection of $K L$ on the hyperplane $\left\{x_{1}=0\right\}$ contains $\left(0, x_{2}, \ldots, x_{d}\right)$, and set $\psi_{K L}(x)=0$ otherwise. One has

$$
\left|\bar{v}^{h}(x)\right|^{p} \leq\left.\frac{1}{2} \sum_{K L L} \psi_{K L}(x)|| v_{L}\right|^{p}-\left|v_{K}\right|^{p} \mid \leq C \sum_{K \mid L} \psi_{K L L}(x) d_{K, L} \frac{\left|v_{L}-v_{K}\right|}{d_{K, L}}\left(\left|v_{K}\right|^{p-1}+\left|v_{L}\right|^{p-1}\right) .
$$

Since $\int_{\Omega} \psi_{K L}(x) d x \leq m(K L) \mathfrak{d}(\Omega)$, one has by the Hölder inequality

$$
\int_{\Omega}\left|\bar{v}^{h}(x)\right|^{p} d x \leq C \mathfrak{d}(\Omega)\left(\sum_{K \mid L} \frac{1}{d} m(K L) d_{K, L}\left|\frac{v_{L}-v_{K}}{d_{K, L}}\right|^{p}\right)^{\frac{1}{p}}\left(\sum_{K L L} \frac{1}{d} m(K L) d_{K, L}\left(\left|v_{K}\right|^{p}+\left|v_{L}\right|^{p}\right)\right)^{\frac{p-1}{p}} .
$$

Denote $h=\operatorname{size}(\mathcal{T})$. Assertion (i) will follow by the Young inequality if we show that

$$
\sum_{K L} \frac{1}{d} m(K L) d_{K, L}\left(\left|v_{K}\right|^{p}+\left|v_{L}\right|^{p}\right) \leq\left(1+2^{p}\right) \sum_{K} \mathfrak{m}(K)\left|v_{K}\right|^{p}+2(2 h)^{p} \sum_{K L L} \frac{1}{d} m(K L) d_{K, L}\left|\frac{v_{L}-v_{K}}{d_{K, L}}\right|^{p},
$$

since $h \leq \mathfrak{d}(\Omega)$. Denote by $R$ the left-hand side of (2.13). We have $d_{K, L}=d_{K, K L L}+d_{L, K \mid L}$; thus

$$
R=\sum_{K} \mathfrak{m}(K)\left|v_{K}\right|^{p}+\sum_{K L} \frac{1}{d} m(K \mid L)\left(\left|v_{K}\right|^{p} d_{L, K L}+\left|v_{L}\right|^{p} d_{K, K L L}\right) .
$$

Note that

$$
\left|v_{K}\right|^{p} d_{L, K L} \leq \begin{cases}2^{p}\left|v_{L}\right|^{p} d_{L, K L} & \text { if }\left|v_{K}\right| \leq 2\left|v_{L}\right| \\ (2 h)^{p}\left|\frac{v_{L}-v_{K}}{d_{K, L}}\right|^{p} d_{K, L} & \text { otherwise. }\end{cases}
$$

Indeed, if $\left|v_{K}\right|>2\left|v_{L}\right|$, one has $\left|v_{L}-v_{K}\right|>\frac{1}{2}\left|v_{K}\right|$ so that

$$
\left|v_{K}\right|^{p} d_{L, K L} \leq\left|v_{K}\right|^{p} d_{K, L} \leq 2^{p}\left|v_{L}-v_{K}\right|^{p} d_{K, L} \leq 2^{p} h^{p}\left|\frac{v_{L}-v_{K}}{d_{K, L}}\right|^{p} d_{K, L} .
$$

Using the same argument for $\left|v_{L}\right|^{p} d_{K, K L}$, we obtain the desired estimate (2.13).

(ii) Now for $x \in \mathbb{R}^{d}$, set $\bar{\psi}_{K \mid L}(x)=1$ in the case where the segment $[x, x+\Delta x]$ crosses $K L$, and set $\bar{\psi}_{K L}(x)=0$ otherwise. Note that $\int_{\Omega} \bar{\psi}_{K L}(x) d x \leq m(K L) \Delta$; hence (ii) follows, since

$$
\int_{\mathbb{R}^{d}}\left|\bar{v}^{h}(x)-\bar{v}^{h}(x+\Delta x)\right| d x \leq \int_{\mathbb{R}^{d}} \sum_{K \mid L} \bar{\psi}_{K \mid L}(x)\left|v_{L}-v_{K}\right| d x \leq \Delta \sum_{K \mid L} m(K \mid L) d_{K, L}\left|\frac{v_{L}-v_{K}}{d_{K, L}}\right| .
$$


(iii) Proceeding as in (ii), we get

$$
\int_{\mathbb{R}^{d}}\left|\bar{v}^{h}(x)-\bar{v}^{h}(x+\Delta x)\right|^{p} d x \leq \int_{\mathbb{R}^{d}}\left(\sum_{K \mid L} \bar{\psi}_{K L}(x) d_{K, L}\right)^{p-1}\left(\sum_{K \mid L} \bar{\psi}_{K \mid L}(x) d_{K, L}\left|\frac{v_{L}-v_{K}}{d_{K, L}}\right|^{p}\right) d x .
$$

It suffices to prove that

$$
\sup _{x, \Delta x \in \Omega,|\Delta x|<\Delta} \sum_{K L} \bar{\psi}_{K L}(x) d_{K, L} \leq \widetilde{C}(\Delta+2 \operatorname{size}(\mathcal{T}))
$$

with some constant $\widetilde{C}$ which depends on $\zeta_{*}(\mathcal{T})$. Recall that $\operatorname{size}(\mathcal{T})$ is the maximum diameter of $K \in \mathcal{T}$ and each mesh contains a ball of the radius $\zeta_{*}(\mathcal{T}) \operatorname{size}(\mathcal{T})$. Denote $h=\operatorname{size}(\mathcal{T})$. Let $\mathcal{Z}_{x, x+\Delta x}$ be the cylinder in $\mathbb{R}^{d}$ of radius $h$, with the segment $\left[x-h \frac{\Delta x}{\Delta x}, x+\Delta x+h \frac{\Delta x}{\mid \Delta x}\right]$ for the axis. If the segment $[x, x+\Delta x]$ crosses a control volume $K, K$ is contained in $\mathcal{Z}_{x, x+\Delta x}$. Note that the $d$-dimensional measure of $\mathcal{Z}_{x, x+\Delta x}$ is estimated by $C(|\Delta x|+2 h) h^{d-1}$. On the other hand, for all $K, \mathfrak{m}(K) \geq C\left(\zeta_{*} h\right)^{d}$, and the greatest possible value of $\sum_{L \in \mathcal{N}_{K}} \bar{\psi}_{K L}(x) d_{K, L}$ is $2 h$. Therefore the left-hand side of (2.14) is estimated by $2 h$ times the number of control volumes contained in $\mathcal{Z}_{x, x+\Delta x}$, i.e., by $2 h \frac{(|\Delta x|+2 h) h^{d-1}}{\left(\zeta_{*}(\mathcal{T}) h\right)^{d}}$, which concludes the proof.

Remark 2 Calculating more carefully $\int_{\Omega} \bar{\psi}_{K L}(x)$ (cf. e.g. [15, 17]), we can prove estimate (iii) in case $1<p \leq 2$ with a constant independent of $\zeta_{*}$.

Now we can state the result for existence and uniqueness of a discrete solution.

Theorem 1 (Existence and uniqueness) Let $\mathcal{T}^{h}$ be a finite volume mesh of $\Omega, k^{h}>0$, and let $\mathcal{D}^{h}$ be a discrete gradient associated to $\mathcal{T}$. If $\mathcal{D}^{h}$ satisfies (4iii), there exists a solution $\left(v_{K}^{n}\right)_{K, n}$ to the discrete problem $\left(\mathcal{S}^{h}\right)$. If $\mathcal{D}^{h}$ satisfies (4ii), the solution is unique.

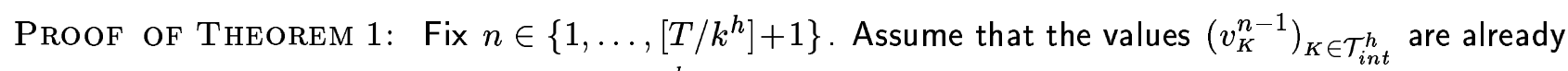
found. We denote by $\mathcal{V}$ a vector of $\mathbb{R}^{\operatorname{card}\left(\mathcal{T}_{\text {int }}^{h}\right)}$ with entries $\left(v_{K}^{n}\right)_{K \in \mathcal{T}_{\text {int }}^{h}}$. Let us consider the operator $\mathcal{S}$ that associates to a given vector $\mathcal{V}$ the vector given by $(2.9)$,

$$
\mathcal{S}(\mathcal{V})=\left(\mathfrak{m}(K) \frac{b\left(v_{K}^{n}\right)-b\left(v_{K}^{n-1}\right)}{k^{h}}-\sum_{L \in \mathcal{N}_{K}} \int_{K L} a_{p}\left(D_{K L}^{n}(x)\right) d x \cdot \nu_{K, L}\right)_{K \in \mathcal{T}_{i n t}^{h}} .
$$

We are looking for a solution to the equation $\mathcal{S}(\mathcal{V})=0$. Consider the scalar product $(\mathcal{S}(\mathcal{V}), \mathcal{V})$ in $\mathbb{R}^{\operatorname{card}\left(\mathcal{T}_{\text {int }}^{h}\right)}$. We have

$$
\begin{aligned}
(\mathcal{S}(\mathcal{V}), \mathcal{V})=\frac{1}{k^{h}} \sum_{K} \mathfrak{m}(K) & b\left(v_{K}^{n}\right) v_{K}^{n}-\frac{1}{k^{h}} \sum_{K} \mathfrak{m}(K) b\left(v_{K}^{n-1}\right) v_{K}^{n}- \\
& -\sum_{K L} v_{K}^{n} \int_{K L} a_{p}\left(D_{K L}^{n}(x)\right) d x \cdot \nu_{K, L}
\end{aligned}
$$

In view of hypothesis (1.2), the first term on the left-hand side of (2.15) is non-negative. Since all the norms are equivalent on $\mathbb{R}^{c a r d\left(\mathcal{T}_{\text {int }}^{h}\right)}$, for the second term on the left-hand side of $(2.15)$ we have

$$
\frac{1}{k^{h}} \sum_{K} \mathfrak{m}(K) b\left(v_{K}^{n-1}\right) v_{K}^{n} \leq|\mathcal{V}| \frac{1}{k^{h}} \sum_{K} \mathfrak{m}(K) b\left(v_{K}^{n-1}\right)=C|\mathcal{V}|
$$

here and in the sequel of the proof, $C, c$ denote positive constants independent of $\mathcal{V}$, and $|\mathcal{V}|$ denotes a norm of $\mathcal{V}$. We then handle the last term on the left-hand side of $(2.15)$, which we denote by $\mathcal{R}(\mathcal{V})$. Using Remark 1, we get

$$
-\mathcal{R}(\mathcal{V})=\sum_{K \mid L}\left(v_{L}^{n}-v_{K}^{n}\right) \int_{K L} a_{p}\left(D_{K \mid L}^{n}(x)\right) d x \cdot \nu_{K, L}
$$


Thanks to hypothesis (4 iii) and Lemma $1(\mathrm{i})$, we obtain

$$
-\mathcal{R}(\mathcal{V}) \geq c \sum_{K L} d_{K, L} m(K \mid L)\left|\frac{v_{L}^{n}-v_{K}^{n}}{d_{K, L}}\right|^{p} \geq c \sum_{K} \mathfrak{m}(K)\left|v_{K}^{n}\right|^{p} \geq c|\mathcal{V}|^{p} .
$$

Returning to (2.15), we obtain for all $1<p<\infty$ that there exist constants $c, C>0$ such that

$$
(\mathcal{S}(\mathcal{V}), \mathcal{V}) \geq c|\mathcal{V}|^{p}-C|\mathcal{V}| \geq 0
$$

for $|\mathcal{V}|$ large enough. Therefore in view of the Brouwer fixed point theorem (cf., e.g., [25, Lemme 4.3]), there exists a solution to $\mathcal{S}(\mathcal{V})=0$.

Uniqueness is easily obtained by induction in $n \in\left\{1, \ldots,\left[T / k^{h}\right]+1\right\}$, due to the monotonicity of $b(\cdot)$ and the strict monotonicity (4ii) of the scheme.

\subsection{INTERPOLATION PROPERTY AND MAIN RESULT}

Consider a family of finite volume schemes $\left(\left(\mathcal{T}^{h}, k^{h}, \mathcal{D}^{h}\right)\right)_{h}$ such that $h$ tends to 0 . Let $\left(v_{K}^{n}\right)_{K, n}$ be a solution to the scheme $\left(\mathcal{S}^{h}\right)$ and $\bar{v}^{h}$ the corresponding discrete solution.

We require the existence of what will be called "interpolated solutions" for $\bar{v}^{h}$, denoted by $v^{h}$, such that $v^{h} \in E$; these should be close to $\bar{v}^{h}$ (asymptotically as $h \rightarrow 0$ ) and satisfy the a priori estimate in $E$ analogous to the estimate of Proposition $1(\mathrm{i})$ on $\bar{v}^{h}$. Moreover, the values $\left(v_{K}^{n}\right)_{K, n}$ should be recoverable from $v^{h}$. To this end, we require $\mathcal{M}^{h}\left[v^{h}\right]=\left(v_{K}^{n}\right)_{K, n}$.

Definition 5 (Interpolation property) A family of grids $\left(\mathcal{T}^{h}, k^{h}\right)_{h}$ has the interpolation property in $E$ if, for any family $\left(\bar{v}^{h}\right)_{h}$ of functions such that $\left.\bar{v}^{h}\right|_{Q_{K}^{n}}=v_{K}^{n} \equiv$ const for each $K \in \mathcal{T}^{h}, n \in\left\{1, \ldots,\left[T / k^{h}\right]+1\right\}$ with $v_{K}^{n}=0$ for $K \in \mathcal{T}_{e x t}^{h}$ and with $\left\|\mathcal{D}_{\perp}^{h}\left[\bar{v}^{h}\right]\right\|_{L^{p}(Q)} \leq C$ for all $h$, there exists a family $\left(v^{h}\right)_{h} \subset E$ such that

$$
\begin{gathered}
\left\|v^{h}-\bar{v}^{h}\right\|_{L^{p}(Q)} \rightarrow 0 \quad \text { as } h \rightarrow 0, \\
\mathcal{M}^{h}\left[v^{h}\right]=\left(v_{K}^{n}\right)_{K, n}, \\
\left\|v^{h}\right\|_{E} \leq I(C) \quad \text { with some function } I: \mathbb{R}^{+} \mapsto \mathbb{R}^{+} \text {independent of } h .
\end{gathered}
$$

If $\bar{v}^{h}$ is a solution to a finite volume scheme, we say that $v^{h}$ is an interpolated solution for $\bar{v}^{h}$.

The interpolation property is the main technical assumption required by the "continuous" approach. In Section 5.2 we give two conditions ensuring this property.

We are now in position to state the main result of this paper.

Theorem 2 (Convergence) Let $\left(\left(\mathcal{T}^{h_{m}}, k^{h_{m}}, \mathcal{D}^{h_{m}}\right)\right)_{m \in \mathbb{N}}$ be a sequence of finite volume schemes, where $k^{h_{m}}+\operatorname{size}\left(\mathcal{T}^{h_{m}}\right) \leq h_{m} \rightarrow 0$ as $m \rightarrow \infty$. Assume that the family of meshes is weakly proportional, the gradient approximation is admissible, and the interpolation property holds (cf. Definitions 3, 4 and 5).

For $m \in \mathbb{N}$, let $\bar{v}^{h_{m}}$ be a discrete solution of $\left(S^{h_{m}}\right)$. Then there exists a subsequence $\left(h_{m_{l}}\right)_{l \in \mathbb{N}}$ such that $\bar{v}^{h_{m_{l}}} \rightarrow v$ in $L^{p}(Q)$ as $l \rightarrow \infty$, where $v$ is a weak solution of Problem (1.1). 
Note that it suffices to strengthen slightly assumption (4ii) of Definition 4 in order to get the strong convergence of $\bar{v}^{h_{m}}$ to $v$ in $L^{p}(Q)$ (cf. Corollary 1 in Section 4). Moreover, in the case where $N=1$ the whole sequence converges to the unique solution of (1.1). In this case error estimates can be proved (cf., e.g., [15] for the linear case), but this is not the purpose of the present paper.

In what follows, we write $k$ instead of $k^{h}$, omit subscripts in sequences $\left(h_{m}\right),\left(h_{m_{l}}\right)$ and simply write that $h$ tends to zero.

\section{The "CONTINUOUS" APPROACH}

Take the discrete solution $\bar{v}^{h}=\sum_{K, n} v_{K}^{n} \mathbb{1}_{Q_{K}^{n}}$ produced by the finite volume scheme $\left(\mathcal{S}^{h}\right)$. Let $v^{h} \in E$ be a corresponding interpolated solution. We will show that there exist functions $u^{h} \in L^{1}(Q)$ and $G^{h} \in L^{p}(Q)$ such that $u^{h}(0, \cdot)=u_{0}^{h}(\cdot)$ and $u^{h}{ }_{t}=\operatorname{div} a_{p}\left(G^{h}\right)$ in the weak sense of Definition 1 , and the functions $u^{h}, G^{h}$ can be recovered from the interpolated solution. More exactly, we prove in Proposition 2 below that $u_{t}^{h} \in \mathcal{D}^{\prime}$ can be extended to $\chi^{h} \in E^{\prime}$ and

$$
<\chi^{h}, \phi>_{E^{\prime}, E}+\iint_{Q} a_{p}\left(\mathcal{G}^{h}\left[v^{h}\right]\right) \cdot D \phi=0 \quad \text { for all } \phi \in E,
$$

with an operator $\mathcal{G}^{h}: E \mapsto L^{p}(Q)$ to be defined, and

$$
<\chi^{h}, \xi>_{E^{\prime}, E}=-\iint_{Q} u^{h} \xi_{t}-\int_{\Omega} u_{0}^{h}(\cdot) \xi(0, \cdot) \quad \begin{aligned}
& \text { for all } \xi \in E \text { with } \\
& \xi_{t} \in L^{\infty}(Q), \xi(T, \cdot)=0 .
\end{aligned}
$$

The analogy of (3.20),(3.21) with (1.4),(1.5) in Definition 1 plays the key role in the proof of the convergence result of Theorem 2.

\subsection{INTERPol ATED GRADIENT AND THE "CONTINUOUS" FORM OF THE SCHEME}

First define $u^{h}$ as the piecewise affine in $t$ interpolation of $b\left(\bar{v}^{h}\right)$ :

$$
u^{h}=\sum_{K, n}\left(b\left(v_{K}^{n}\right)+\frac{t-k n}{k}\left(b\left(v_{K}^{n}\right)-b\left(v_{K}^{n-1}\right)\right)\right) \mathbb{1}_{Q_{K}^{n}} .
$$

Then (3.21) holds, since $u^{h}(0, \cdot)=u_{0}^{h}(\cdot)$ and the piecewise constant function $u^{h}{ }_{t}$ extends to $\chi^{h} \in E^{\prime}$ by

$$
<\chi^{h}, \phi>_{E^{\prime}, E}=\iint_{Q} u^{h} \phi \quad \text { for all } \phi \in E .
$$

Next, note that in $\left(\mathcal{S}^{h}\right)$ the numerical flux is prescribed on the boundary of each control volume; we will extend it to $Q$ as follows. For given $K \in \mathcal{T}_{\text {int }}^{h}, n$ and a function $F_{K}^{n}: \partial K \mapsto \mathbb{R}$, consider the following Neumann problem in the factor space $\mathcal{W}(K)=W^{1, p}(K) / \mathbb{R}$ :

$$
\left\{\begin{array}{l}
\operatorname{div} a_{p}(D w)=\frac{1}{\mathfrak{m}(K)} \sum_{K \in \mathcal{N}_{K}} \int_{K L} F_{K}^{n} \quad \text { on } \quad K \\
a_{p}(D w) \cdot \nu_{K} \mid \partial_{K}=F_{K}^{n},
\end{array}\right.
$$

where $\nu_{K}$ is the exterior unit normal vector to $\partial K$. For $K \in \mathcal{T}_{\text {ext }}^{h}$ with $\mathfrak{m}(K)>0$ we drop in (3.24) the Neumann boundary condition on $\partial K \cap \partial \Omega$ and seek $w \in W^{1, p}(K)$ with $\left.w\right|_{\partial K \cap \partial \Omega}=0$. 
Lemma 2 Let $F_{K}^{n} \in L^{p^{\prime}}(\partial K)\left(F_{K}^{n} \in L^{p^{\prime}}(\partial K \backslash \partial \Omega)\right.$, if $\left.{ }_{K} \in \mathcal{T}_{e x t}^{h}\right)$. Then (3.24) admits a unique solution. Proof : Let us give the proof for $K \in \mathcal{T}_{\text {int }}^{h}$. Supply $\mathcal{W}$ with the norm $\|w\|_{\mathcal{W}}=\left(\int_{K}|D w|^{p}\right)^{1 / p}$ (we will ambiguously denote by the same symbol an element of $W^{1, p}(K)$ and the corresponding equivalence class as element of $\mathcal{W})$. Consider the operator $A: w \in \mathcal{W} \mapsto A[w] \in \mathcal{W}^{\prime}$ defined by

$$
<A[w], \varphi>_{\mathcal{W}^{\prime}, \mathcal{W}}=\int_{K} a_{p}(D w) \cdot D \varphi
$$

for all $\varphi \in \mathcal{W}$, and the functional $f \in \mathcal{W}^{\prime}$ defined by

$$
<f, \varphi>_{\mathcal{W}^{\prime}, \mathcal{W}}=-\frac{1}{\mathfrak{m}(K)} \int_{K} \varphi \int_{\partial K} F_{K}^{n}+\int_{\partial K} \varphi F_{K}^{n},
$$

for all $\varphi \in \mathcal{W}$. Note that $f$ is well-defined, since the right-hand side of (3.26) is invariant under translation by a constant in $W^{1, p}(K)$.

The operator $A$ in (3.25) is bounded, hemicontinuous, strictly monotone on $\mathcal{W}$, and

$$
\frac{<A[w], w>_{\mathcal{W}^{\prime}, \mathcal{W}}}{\|w\|_{\mathcal{W}}}=\frac{\int_{K}|D w|^{p}}{\|w\|_{\mathcal{W}}}=\|w\|_{\mathcal{W}}^{p-1} \rightarrow \infty
$$

as $\|w\|_{\mathcal{W}} \rightarrow \infty$. Thus $A$ is bijective (cf., e.g., [25, Chapitre 2, Théorème 2.1]); we have $A[w]=f$ for a unique $w \in \mathcal{W}$, which was our claim.

The case $K \in \mathcal{T}_{\text {ext }}^{h}$ is similar, using in the place of $\mathcal{W}$ the space of functions of $W^{1, p}(K)$ with zero trace on $\partial_{K} \cap \partial \Omega$.

The function $G^{h}$, which can be understood as a sort of gradient of $\bar{v}^{h}$, is defined by

$$
\left\{\begin{array}{l}
G^{h}=\sum_{K, n} D w_{K}^{n} \mathbb{1}_{Q_{K}^{n}}^{n}, \quad \text { where } \quad w_{K}^{n} \text { solves }(3.24) \text { with } \\
F_{K}^{n}(x)=a_{p}\left(D_{K L}^{n}(x)\right) \cdot \nu_{K} \quad \text { for } \quad x \in K L \in \mathcal{E}_{K} \text { and }\left(D_{K L}^{n}\right)_{K, n}=\mathcal{D}^{h}\left[\left(v_{K}^{n}\right)_{K, n}\right] .
\end{array}\right.
$$

More generally, let us give the following definition.

Definition 6 (Interpolated gradient operator) The interpolated gradient operator $\mathcal{G}^{h}: E \mapsto L^{p}(Q)$ maps $\eta \in E$ into $\mathcal{G}^{h}[\eta]$ given by

$$
\left\{\begin{array}{l}
\mathcal{G}^{h}[\eta]=\sum_{K, n} D \eta_{K}^{n} \mathbb{1}_{Q_{K}^{n}}, \quad \text { where } \eta_{K}^{n} \in \mathcal{W}(K) \text { solves } \\
-\int_{K} a_{p}\left(D \eta_{K}^{n}\right) \cdot D \varphi+\sum_{L \in \mathcal{N}_{K}} \int_{K L} \varphi a_{p}\left(D_{K L}^{n}\right) \cdot \nu_{K}=\frac{1}{\mathfrak{m}(K)} \int_{K} \varphi \sum_{L \in \mathcal{N}_{K}} \int_{K L} a_{p}\left(D_{K L}^{n}\right) \cdot \nu_{K} \\
\text { for all } \varphi \in W^{1, p}(K) \quad\left(\text { for all } \varphi \in W^{1, p}(K) \text { with }\left.\varphi\right|_{\partial K \cap \partial \Omega}=0, \text { in case } K \in \mathcal{T}_{\text {ext }}^{h}\right) \\
\text { and the values } D_{K L}^{n}(x) \text { are given by }\left(D_{K L}^{n}\right)_{K L, n}=\mathcal{D}^{h}[\eta] .
\end{array}\right.
$$

We remark that $G^{h}=\mathcal{G}^{h}\left[v^{h}\right]$ by property (2.18) of interpolated solutions $v^{h}$. We show that (3.20) follows from (3.23) and the conservation of fluxes.

Proposition 2 (The continuous form of the scheme) Assume that $\left(v_{K}^{n}\right)_{K, n}$ is a solution of $\left(\mathcal{S}^{h}\right)$. Let $v^{h}$ be a corresponding interpolated solution, let $u^{h}$ and $\chi^{h}$ be defined by (3.22) and (3.23), respectively, and let $\mathcal{G}^{h}$ be the interpolated gradient operator of Definition 6. Then (3.20),(3.21) hold. 
Proof : It remains to check (3.20). By (3.22), for all $K \in \mathcal{T}^{h}$ and $n$, we have

$$
u^{h}{ }_{t}-\operatorname{div} a_{p}\left(G^{h}\right)=\frac{b\left(v_{K}^{n}\right)-b\left(v_{K}^{n-1}\right)}{k}-\frac{1}{\mathfrak{m}(K)} \sum_{L \in \mathcal{N}_{K}} \int_{K L} a_{p}\left(D_{K \mid L}^{n}(x)\right) d x \cdot \nu_{K, L}=0
$$

everywhere on $Q_{K}^{n}$ because of (2.9). Therefore, using (3.23) and integrating by parts in each $Q_{K}^{n}$, we have

$$
\begin{aligned}
<\chi^{h}, \phi>_{E^{\prime}, E}+\int_{Q} a_{p}\left(\mathcal{G}^{h}\left[v^{h}\right]\right) \cdot D \phi=\iint_{Q} u^{h}{ }_{t} \phi+a_{p}\left(G^{h}\right) \cdot D \phi=\sum_{K, n} \iint_{Q_{K}^{n}}\left(u^{h}{ }_{t}-\operatorname{div} a_{p}\left(G^{h}\right)\right) \phi+ \\
\quad+\sum_{K, n} \sum_{L \in \mathcal{N}_{K}} \iint_{I^{n} \times K L} \phi a_{p}\left(D_{K \mid L}^{n}\right) \cdot \nu_{K, L}=0+\sum_{K L, n} \iint_{I^{n} \times K L} \phi a_{p}\left(D_{K \mid L}^{n}\right) \cdot\left(\nu_{K, L}+\nu_{L, K}\right)=0 .
\end{aligned}
$$

\subsection{Properties of the INTERPolated GRAdient AND CONSISTENCY}

In view of (3.20) and (1.4), it is natural to compare the elliptic operator in (1.1),

$$
\mathcal{A}: \eta \in E \mapsto-\operatorname{div} a_{p}(D \eta) \in E^{\prime},
$$

with the operators

$$
\mathcal{A}^{h}: \eta \in E \mapsto-\operatorname{div} a_{p}\left(\mathcal{G}^{h}[\eta]\right) \in E^{\prime} .
$$

Indeed, $\mathcal{A}^{h}$ can be considered as the finite volume approximates of $\mathcal{A}$, whence the following definition.

Definition 7 (Consistency of approximation) Let $\left(\left(\mathcal{T}^{h}, k^{h}, \mathcal{D}^{h}\right)\right)_{h}$ be a family of finite volume schemes for Problem (1.1), with $\operatorname{size}\left(\mathcal{T}^{h}\right)+k^{h} \leq h \rightarrow 0$. We say that the approximation of (1.1) by these schemes is consistent if, for all $\eta \in E$, one has $\mathcal{A}^{h}[\eta] \rightarrow A[\eta]$ in $E^{\prime}$ as $h \rightarrow 0$.

In this section we prove the following result.

Theorem 3 (Consistency) Let $\left(\left(\mathcal{T}^{h}, k^{h}, \mathcal{D}^{h}\right)\right)_{h}$ be a family of finite volume schemes with a weakly proportional family of meshes and an admissible gradient approximation (cf. Definitions 3 and 4). Then it provides a consistent approximation of (1.1), in the sense of Definition 7.

The proof of Theorem 3 is based upon the following properties of the interpolated gradient operator $\mathcal{G}^{h}$. Proposition 3 Let $\left(\left(\mathcal{T}^{h}, k^{h}, \mathcal{D}^{h}\right)\right)_{h}$ be a family of finite volume schemes with admissible gradient approximation and weakly proportional family of meshes.

(i) There exists a constant $C$ such that for all $\eta \in E$ and $H \subset Q$ such that $H=\bigcup_{i=1}^{m} Q_{K_{i}}^{n_{i}}$,

$$
\iint_{H}\left|\mathcal{G}^{h}[\eta]\right|^{p} \leq C \iint_{\Upsilon_{\varsigma+1}(H)}|D \eta|^{p}
$$

where $\Upsilon_{\varsigma+1}(H)=\bigcup_{i=1}^{m} I^{n_{i}} \times \Upsilon_{\varsigma+1}\left(K_{i}\right)$. In particular, $\left(\mathcal{G}^{h}\right)_{h}$ are uniformly bounded on $E$ and

$$
\left\|\mathcal{G}^{h}[\eta]\right\|_{L^{p}(Q)} \leq C\|\eta\|_{E} \text {. }
$$

(ii) The operators $\left(\mathcal{G}^{h}\right)_{h}$ are locally equicontinuous on $E$. More exactly, there exists a constant $C(R)$ such that

$$
\begin{gathered}
\left\|\mathcal{G}^{h}[\eta]-\mathcal{G}^{h}[\mu]\right\|_{L^{p}(Q)} \leq C(R)\left(\|\eta-\mu\|_{E}\right)^{\alpha} \\
\left\|a_{p}\left(\mathcal{G}^{h}[\eta]\right)-a_{p}\left(\mathcal{G}^{h}[\mu]\right)\right\|_{L^{p^{\prime}(Q)}} \leq C(R)\left(\|\eta-\mu\|_{E}\right)^{\beta}
\end{gathered}
$$

whenever $\|\eta\|_{E} \leq R$ and $\|\mu\|_{E} \leq R$, where $\alpha=\min \left\{p-1, \frac{1}{p-1}\right\}, \beta=\min \left\{(p-1)^{2}, \frac{1}{p-1}\right\}$. 
In the statement above and in the rest of this section, $C$ denotes a generic constant that depends only on $p, d, \Omega$, on $M, \zeta^{*}$ of (2.7) and on $C_{*}, C^{*}, \varsigma$ of Definition 4, unless the additional dependence on $R$ is specified. The proof uses the standard properties of the function $a_{p}(\cdot)$ (cf. $\left.[19,12]\right)$ : for all $y_{1}, y_{2} \in \mathbb{R}^{d}$,

$$
\begin{aligned}
& \begin{cases}\left|a_{p}\left(y_{1}\right)-a_{p}\left(y_{2}\right)\right|^{p^{\prime}} \leq C\left|y_{1}-y_{2}\right|^{p}, & 1<p \leq 2 ; \\
\left|a_{p}\left(y_{1}\right)-a_{p}\left(y_{2}\right)\right|^{p^{\prime}} \leq C\left|y_{1}-y_{2}\right|^{p^{\prime}}\left(\left|y_{1}\right|^{p}+\left|y_{2}\right|^{p}\right)^{\frac{p-2}{p-1}}, & p \geq 2 ;\end{cases} \\
& \begin{cases}\left|y_{1}-y_{2}\right|^{p} \leq C\left[\left(a_{p}\left(y_{1}\right)-a_{p}\left(y_{2}\right)\right) \cdot\left(y_{1}-y_{2}\right)\right]^{\frac{p}{2}}\left[\left|y_{1}\right|^{p}+\left|y_{2}\right|^{p}\right]^{\frac{2-p}{2}}, & 1<p \leq 2 ; \\
\left|y_{1}-y_{2}\right|^{p} \leq C\left(a_{p}\left(y_{1}\right)-a_{p}\left(y_{2}\right)\right) \cdot\left(y_{1}-y_{2}\right), & p \geq 2 .\end{cases}
\end{aligned}
$$

Before turning to the proofs of Proposition 3 and Theorem 3, note the following three lemmas.

Lemma 3 Let $K \subset \mathbb{R}^{d}$ be a bounded convex domain of $\mathbb{R}^{d}$ of diameter $\mathfrak{d}(K)$. Assume that $K$ contains a ball $U$ of radius $\zeta^{*} \mathfrak{d}(K)>0$. Then there exists a constant $C$ such that, assigning $\bar{w}=\frac{1}{\mathfrak{m}(V)} \int_{V} w$ for a set $V$ of $d$-dimensional measure $\mathfrak{m}(V)$ such that $U \subset V \subset K$, one has

$$
\text { (i) } \int_{K}|w-\bar{w}|^{p} \leq C(\mathfrak{d}(K))^{p} \int_{K}|D w|^{p}, \quad \text { (ii) } \quad \int_{\partial_{K}}|w-\bar{w}|^{p} \leq C(\mathfrak{d}(K))^{p-1} \int_{K}|D w|^{p}
$$

for all $w \in W^{1, p}(K)$, where $\left.w\right|_{\partial_{K}}$ is understood in the sense of traces.

Proof : Applying, e.g., the proofs of [13, Theorems 59,60,76] with $p=2$ replaced by general $p \in$ $(1,+\infty)$, we obtain the claim of the lemma with $C$ depending on $p, d$ and (in the inequality (ii)) on the Lipschitz continuity of $\partial K$. Due to the convexity of $K, C$ actually depends only on $p, d$, and $\zeta^{*}$. $\diamond$

Lemma 4 Let $\left(\mathcal{T}^{h}\right)_{h}$ be a weakly proportional family of meshes, and let $\left(\mathcal{D}_{\perp}^{h}\right)_{h}$ be the operators defined by (2.12). Then there exists a constant $C$ such that for all $K, n$, for all $\eta \in E$,

$$
\iint_{Q_{K}^{n}}\left|\mathcal{D}_{\perp}^{h}[\eta]\right|^{p} \leq C \iint_{I^{n_{\times} \Upsilon_{1}(K)}}|D \eta|^{p} .
$$

ProOF : Let $\left(\eta_{K}^{n}\right)_{K, n}=\mathcal{M}^{h}[\eta]$ and $\eta_{K L}^{n}=\frac{1}{k m(K L)} \iint_{I^{n} \times K L} \eta$ in the sense of traces. By definition,

$$
\begin{aligned}
\iint_{Q_{K}^{n}}\left|\mathcal{D}_{\perp}^{h}[\eta]\right|^{p}=\sum_{K \mid L} \frac{1}{d} k m(K \mid L) d_{K, K L}\left|\frac{\eta_{L}^{n}-\eta_{K}^{n}}{d_{K, L}}\right|^{p} \leq \\
\leq C \sum_{K \mid L} \frac{1}{d} k m(K \mid L) d_{K, K \mid L}\left(\frac{\left|\eta_{K \mid L}^{n}-\eta_{K}^{n}\right|^{p}}{\left(d_{K, K \mid L}\right)^{p}}+\frac{\left|\eta_{K L}^{n}-\eta_{L}^{n}\right|^{p}}{d_{K, K \mid L}\left(d_{L, K \mid L}\right)^{p-1}}\right) \leq \\
\quad \leq C \sum_{K \mid L} \frac{1}{d} k m(K L) d_{K, K L}\left|\frac{\eta_{K \mid L}^{n}-\eta_{K}^{n}}{d_{K, K L}}\right|^{p}+C \sum_{K \mid L} \frac{1}{d} k m(K \mid L) d_{L, K \mid L}\left|\frac{\eta_{K \mid L}^{n}-\eta_{L}^{n}}{d_{L, K \mid L}}\right|^{p} .
\end{aligned}
$$

By the convexity of the function $z \mapsto|z|^{p}$ and Lemma 3,

$$
k m(K L)\left|\eta_{K L}^{n}-\eta_{K}^{n}\right|^{p} \leq \iint_{I^{n} \times K L}\left|\eta-\eta_{K}^{n}\right|^{p} \leq C \mathfrak{d}(K)^{p-1} \iint_{Q_{K}^{n}}|D \eta|^{p},
$$

and the same holds if $K$ and $L$ are exchanged. Hence by (2.7) we have

$$
\iint_{Q_{K}^{n}}\left|\mathcal{D}_{\perp}^{h}[\eta]\right|^{p} \leq C \sum_{L \in \mathcal{N}_{K}}\left(\iint_{Q_{K}^{n}}|D \eta|^{p}+\iint_{Q_{L}^{n}}|D \eta|^{p}\right) \leq C \iint_{I^{n_{\times} \Upsilon_{1}(K)}}|D \eta|^{p} .
$$


Lemma 5 Let $\left(\left(\mathcal{T}^{h}, k^{h}, \mathcal{D}^{h}\right)\right)_{h}$ be a family of finite volume schemes with a weakly proportional family of meshes and an admissible gradient approximation. Then the following hold.

(i) For all $R>0$ there exists a constant $C(R)$ such that

$$
\sum_{K, n} \mathfrak{d}(K) \iint_{\Sigma_{K}^{n}}\left|a_{p}\left(\mathcal{D}^{h}[\eta]\right)-a_{p}\left(\mathcal{D}^{h}[\mu]\right)\right|^{p^{\prime}} \leq C(R)\left(\|\eta-\mu\|_{E}\right)^{\min \left\{p, p^{\prime}\right\}}
$$

whenever $\|\eta\|_{E} \leq R$ and $\|\mu\|_{E} \leq R$.

(ii) There exists a constant $C$ such that for all $\eta \in E$ and $H, \Upsilon_{\varsigma+1}(H)$ as in Proposition 3 one has

$$
\sum_{i=1}^{m} \mathfrak{d}\left(K_{i}\right) \iint_{\Sigma_{K_{i}}^{n_{i}}}\left|\mathcal{D}^{h}[\eta]\right|^{p} \leq C \iint_{\Upsilon_{\varsigma+1}(H)}|D \eta|^{p}
$$

Proof : First take $K$ and consider $\varphi^{K}=\left|a_{p}\left(\mathcal{D}^{h}[\eta]\right)-a_{p}\left(\mathcal{D}^{h}[\mu]\right)\right|^{p^{\prime}} \in L^{1}(\partial K)$. Recall that the values of $\mathcal{D}^{h}$ have been extended from $\partial_{K}$ inside $K$ by means of the projection operator $\mathcal{P}^{h}$ (cf. Section 2.2). Hence by (2.7) we have

$$
\mathfrak{d}(K) \int_{\partial K}\left|\varphi^{K}\right|=\mathfrak{d}(K) \sum_{L \in \mathcal{N}_{K}} \int_{K L}\left|\varphi^{K}\right|=d \sum_{L \in \mathcal{N}_{K}} \frac{\mathfrak{d}(K)}{d_{K, K \mid L}} \int_{\widehat{K L} \cap K}\left|\varphi^{K} \circ \mathcal{P}^{h}\right| \leq \frac{d}{\zeta^{*}} \int_{K}\left|\varphi^{K} \circ \mathcal{P}^{h}\right| .
$$

If $1<p \leq 2,(3.35)$ and (3.33) yield

$$
\sum_{K, n} \mathfrak{d}(K) \iint_{\Sigma_{K}^{n}}\left|a_{p}\left(\mathcal{D}^{h}[\eta]\right)-a_{p}\left(\mathcal{D}^{h}[\mu]\right)\right|^{p^{\prime}} \leq C \sum_{K, n} \iint_{Q_{K}^{n}}\left|\mathcal{D}^{h}[\eta]-\mathcal{D}^{h}[\mu]\right|^{p}
$$

In turn, (4 i),(4 iv), Lemma 4 and (2.7) imply that

$$
\begin{aligned}
& \sum_{K, n} \iint_{Q_{K}^{n}}\left|\mathcal{D}^{h}[\eta]-\mathcal{D}^{h}[\mu]\right|^{p} \leq C \sum_{K, n} \iint_{I^{n_{\times \Upsilon_{\varsigma}(K)}}}\left|\mathcal{D}_{\perp}^{h}[\eta-\mu]\right|^{p} \leq \\
& \quad \leq C \sum_{K, n} \iint_{I^{n_{\times} \Upsilon_{\varsigma}(K)}}|D(\eta-\mu)|^{p} \leq C \iint_{Q}|D \eta-D \mu|^{p}=C\left(\|\eta-\mu\|_{E}\right)^{p},
\end{aligned}
$$

which was the claim of (i) for $1<p \leq 2$. Furthermore, remark that (3.36) also holds for $p \geq 2$, in particular with $\eta=0$ or $\mu=0$. Therefore for $p \geq 2$, using (3.35) and then (3.33) and the Hölder inequality, we get

$$
\sum_{K, n} \mathfrak{d}(K) \iint_{\Sigma_{K}^{n}}\left|a_{p}\left(\mathcal{D}^{h}[\eta]\right)-a_{p}\left(\mathcal{D}^{h}[\mu]\right)\right|^{p^{\prime}} \leq C\left(\sum_{K, n} \iint_{Q_{K}^{n}}\left|\mathcal{D}^{h}[\eta]-\mathcal{D}^{h}[\mu]\right|^{p}\right)^{\frac{p^{\prime}}{p}} \times\left(R^{p}\right)^{\frac{p-2}{p-1}}
$$

Thus in case $p \geq 2$ (i) also follows from (3.36).

The proof of (ii) is similar, using the identity $\left|a_{p}(y)\right|^{p^{\prime}}=|y|^{p}$ instead of the inequalities (3.33).

Proof of Proposition 3: Recalling Definition 6, for all $K \in \mathcal{T}_{\text {int }}$ and $n$, denote by $\eta_{K}^{n}$ (respectively, by $\left.\mu_{K}^{n}\right)$ a function in $W^{1, p}(K)$ that solves (3.24) with $F_{K}^{n}(x)=a_{p}\left(D_{K \mid L}^{n}(x)\right) \cdot \nu_{K}$ for $x \in K L \in \mathcal{E}_{K}$, where $\left(D_{K \mid L}^{n}\right)_{K \mid L, n}=\mathcal{D}^{h}[\eta]$ (respectively, $\left(D_{K L}^{n}\right)_{K L, n}=\mathcal{D}^{h}[\mu]$ ). In other words, each of $\eta_{K}^{n}, \mu_{K}^{n}$ verifies the 
integral identity corresponding to (3.24) with all test functions in $W^{1, p}(K)$. Taking for the test function $\left(\eta_{K}^{n}-\mu_{K}^{n}\right)$, subtracting the two identities and integrating in $t \in I^{n}$, we obtain

$$
\begin{aligned}
\iint_{Q_{K}^{n}}\left(a_{p}\left(D \eta_{K}^{n}\right)-a_{p}\left(D \mu_{K}^{n}\right)\right) \cdot\left(D \eta_{K}^{n}-D \mu_{K}^{n}\right)= \\
=\int_{I^{n}} \int_{\partial_{K}}\left(\eta_{K}^{n}-\mu_{K}^{n}-\overline{\eta_{K}^{n}-\mu_{K}^{n}}\right)\left(a_{p}\left(\mathcal{D}^{h}[\eta]\right)-a_{p}\left(\mathcal{D}^{h}[\mu]\right)\right) \cdot \nu_{K}
\end{aligned}
$$

where $\overline{\eta_{K}^{n}-\mu_{K}^{n}}=\frac{1}{\mathfrak{m}(K)} \int_{K} \eta_{K}^{n}-\mu_{K}^{n}$ for a.a. $t \in I^{n}$. Summing over $K, n$, using the Hölder inequality and Lemmas 3(ii) and 5(i), we have from (3.37)

$$
\begin{aligned}
& \iint_{Q}\left(a_{p}\left(\mathcal{G}^{h}[\eta]\right)-a_{p}\left(\mathcal{G}^{h}[\mu]\right)\right) \cdot\left(\mathcal{G}^{h}[\eta]-\mathcal{G}^{h}[\mu]\right) \leq \\
& \leq \sum_{K, n} \int_{I^{n}} \int_{\partial K} \mathfrak{d}(K)^{\frac{-1}{p^{\prime}}}\left|\eta_{K}^{n}-\mu_{K}^{n}-\overline{\eta_{K}^{n}-\mu_{K}^{n}}\right| \times \mathfrak{d}(K)^{\frac{1}{p^{\prime}}}\left|a_{p}\left(\mathcal{D}^{h}[\eta]\right)-a_{p}\left(\mathcal{D}^{h}[\mu]\right)\right| \leq \\
& \leq\left(\sum_{K, n} \int_{I^{n}} \mathfrak{d}(K)^{1-p} \int_{\partial_{K}}\left|\eta_{K}^{n}-\mu_{K}^{n}-\overline{\eta_{K}^{n}-\mu_{K}^{n}}\right|^{p}\right)^{\frac{1}{p}}\left(\sum_{K, n} \mathfrak{d}(K) \iint_{\Sigma_{K}^{n}}\left|a_{p}\left(\mathcal{D}^{h}[\eta]\right)-a_{p}\left(\mathcal{D}^{h}[\mu]\right)\right|^{p^{\prime}}\right)^{\frac{1}{p^{\prime}}} \leq \\
& \leq\left(\sum_{K, n} \iint_{Q_{K}^{n}}\left|D \eta_{K}^{n}-D \mu_{K}^{n}\right|^{p}\right)^{\frac{1}{p}}\|\eta-\mu\|_{E}^{\min \left\{p / p^{\prime}, 1\right\}}=\left\|\mathcal{G}^{h}[\eta]-\mathcal{G}^{h}[\mu]\right\|_{L^{p}(Q)}\|\eta-\mu\|_{E}^{\min \left\{p / p^{\prime}, 1\right\}} .
\end{aligned}
$$

In the same manner, taking $\mu=0$ and using Lemma 5(ii), we get

$$
\iint_{H}\left|\mathcal{G}^{h}[\eta]\right|^{p} \leq C\left(\iint_{H}\left|\mathcal{G}^{h}[\eta]\right|^{p}\right)^{\frac{1}{p}}\left(\iint_{\Upsilon_{\varsigma+1}(H)}|D \eta|^{p}\right)^{\frac{1}{p^{\prime}}}
$$

which proves (i).

Now if $1<p \leq 2$, (3.34), (3.38) and the Hölder inequality yield

$$
\left\|\mathcal{G}^{h}[\eta]-\mathcal{G}^{h}[\mu]\right\|_{L^{p}(Q)}^{p} \leq C\left(\left\|\mathcal{G}^{h}[\eta]-\mathcal{G}^{h}[\mu]\right\|_{L^{p}(Q)}\|\eta-\mu\|_{E}^{\frac{p}{p^{\prime}}}\right)^{\frac{p}{2}}\left(\left\|\mathcal{G}^{h}[\eta]\right\|_{L^{p}(Q)}^{p}+\left\|\mathcal{G}^{h}[\mu]\right\|_{L^{p}(Q)}^{p}\right)^{\frac{2-p}{2}}
$$

Using (3.30), we obtain (3.31) with $\alpha=p-1$. Now (3.32) with $\beta=(p-1)^{2}$ follows by (3.33). If $p \geq 2$, (3.34) and (3.38) readily yield (3.31) with $\alpha=1 /(p-1)$; hence (3.32) with $\beta=1 /(p-1)$ follows by (3.33).

Proof of Theorem 3: We have to prove that $\left\|a_{p}(D \eta)-a_{p}\left(\mathcal{G}^{h}[\eta]\right)\right\|_{L^{p^{\prime}}(Q)} \rightarrow 0$ as $h \rightarrow 0$.

Let us first prove the theorem for the case of $\eta \in E$ that is piecewise constant in $t$ and piecewise affine in $x$. Let $J \subset Q$ be the set of discontinuities of $D \eta$. Clearly, $J$ is of finite $d$-dimensional Hausdorff measure $\mathcal{H}^{d}(J)$.

For $\varsigma$ given in Definition 4, let us introduce $H^{h}=\bigcup_{\left\{K, n \mid I^{n} \times \Upsilon_{\varsigma}(K) \cap J \neq \emptyset\right\}} Q_{K}^{n}$. Note that $\left|H^{h}\right| \leq$ $(\varsigma+1) h \mathcal{H}^{d}(J) \rightarrow 0$ as $h \rightarrow 0$; likewise, $\left|\Upsilon_{\varsigma+1}\left(H^{h}\right)\right| \rightarrow 0$ as $h \rightarrow 0$. Therefore, by Proposition 3(i) we have

$$
\iint_{H^{h}}\left|a_{p}(D \eta)-a_{p}\left(\mathcal{G}^{h}[\eta]\right)\right|^{p^{\prime}} \leq C\left(\iint_{H^{h}}|D \eta|^{p}+\iint_{\Upsilon_{\varsigma+1}\left(H^{h}\right)}|D \eta|^{p}\right) \rightarrow 0
$$

as $h \rightarrow 0$. Moreover, for all $Q_{K}^{n}$ such that $Q_{K}^{n} \cap H^{h}=\varnothing$, we have $\mathcal{G}^{h}[\eta] \equiv D \eta$ on $Q_{K}^{n}$. Indeed, we have $D[\eta] \equiv$ const on $\Upsilon_{\varsigma+1}\left(Q_{K}^{n}\right)$. Therefore $\left.\mathcal{D}^{h}[\eta]\right|_{Q_{K}^{n}} \equiv D \eta=$ const by property (4 iv) of admissible gradient 
approximations. Hence $D w=D \eta$ satisfies the boundary condition in (3.24); the equation is also satisfied, since $\operatorname{div} a_{p}(D \eta) \equiv 0$ on $K$ and $\frac{1}{\mathfrak{m}(K)} \int_{\partial K} a_{p}\left(\mathcal{D}^{h}[\eta]\right) \cdot \nu_{K}=a_{p}(D \eta) \cdot \int_{\partial K} \nu_{K}=0$.

It follows that $\left\|a_{p}(D \eta)-a_{p}\left(\mathcal{G}^{h}[\eta]\right)\right\|_{L^{p^{\prime}}(Q)} \rightarrow 0$ as $h \rightarrow 0$, which was our claim.

Now let us approximate an arbitrary function $\eta$ in $E$ by functions $\eta_{l}$ that are piecewise constant in $t$ and piecewise affine in $x$. Note that we can always choose this sequence $\eta_{l}$ in $E$ such that $\eta_{l} \rightarrow \eta$ in $E$ and a.e. on $Q$ as $l \rightarrow \infty$, and $\left|D \eta_{l}\right|^{p}$ are dominated by an $L^{1}(Q)$ function independent of $l$. We have

$$
\begin{aligned}
\left\|a_{p}(D \eta)-a_{p}\left(\mathcal{G}^{h}[\eta]\right)\right\|_{L^{p^{\prime}}(Q)} \leq\left\|a_{p}(D \eta)-a_{p}\left(D \eta_{l}\right)\right\|_{L^{p^{\prime}}(Q)}+ \\
+\left\|a_{p}\left(D \eta_{l}\right)-a_{p}\left(\mathcal{G}^{h}\left[\eta_{l}\right]\right)\right\|_{L^{p^{\prime}}(Q)}+\left\|a_{p}\left(\mathcal{G}^{h}\left[\eta_{l}\right]\right)-a_{p}\left(\mathcal{G}^{h}[\eta]\right)\right\|_{L^{p^{\prime}}(Q)} .
\end{aligned}
$$

As $l \rightarrow 0$, the first term in the right-hand side of (3.39) converges to zero by the Lebesgue dominated convergence theorem, independently of $h$. The second one converges to zero as $h \rightarrow 0$ for all $l$ fixed. Finally, by Proposition 3(ii), the third one converges to zero as $l \rightarrow \infty$ uniformly in $h$. Hence the result follows.

\section{Proof of TheOREM 2}

In the context of continuous dependence upon the data of weak solutions to "general" elliptic-parabolic problems (cf. [2, Chap.V]), the proof of convergence of weak solutions of approximating problems is based upon the three essential arguments (A), (B) and (C) below.

(A) A priori estimates, using (1.2) and the Alt-Luckhaus chain rule lemma (cf. $[1,26,10])$.

(B) Strong compactness in the parabolic term, using a variant of the Kruzhkov lemma (cf. [23]):

Lemma 6 (cf. [2, Chap.V,Lemma 6]) Let $\Omega$ be an open domain in $\mathbb{R}^{d}, Q=(0, T) \times \Omega$ and let the families of functions $\left(u^{h}\right)_{h},\left(F_{\alpha}^{h}\right)_{h, \alpha}$ be bounded in $L^{1}(Q)$ and satisfy $\frac{\partial}{\partial t} u^{h}=\sum_{|\alpha| \leq m} D^{\alpha} F_{\alpha}^{h}$ in $\mathcal{D}^{\prime}(Q)$. Assume that $u^{h}$ can be extended by zero outside $Q$, and one has

$$
\sup _{|\Delta x| \leq \Delta} \iint_{\mathbb{R}^{d+1}}\left|u^{h}(t, x+\Delta x)-u^{h}(t, x)\right| d x d t \leq \omega(\Delta), \quad \text { with } \lim _{\Delta \rightarrow 0} \omega(\Delta)=0,
$$

where $\omega(\cdot)$ does not depend on $h$. Then $\left(u^{h}\right)_{h}$ is relatively compact in $L^{1}(Q)$.

(C) Convergence in the elliptic term, using a variant of the Minty-Browder argument (cf., e.g., [25]):

Lemma 7 (cf. [2, Chap.V,Lemma 7]) Let $E$ be a Banach space, $E^{\prime}$ its dual and $\langle\cdot, \cdot\rangle$ denote the duality product of elements of $E^{\prime}$ and $E$. Let $\left(v^{h}\right)_{h} \subset E$ and $v^{h} \rightarrow v$ as $h \rightarrow 0$. Let $\mathcal{A}^{h}$ be a sequence of monotone operators from $E$ to $E^{\prime}$ such that $\mathcal{A}^{h}\left[v^{h}\right] \stackrel{*}{\rightarrow}-\chi$ for some $\chi \in E^{\prime}$. Assume that $\mathcal{A}^{h}$ converge pointwise to some operator $\mathcal{A}$, and $\mathcal{A}$ is hemicontinuous (i.e., continuous in the weak-* topology of $E^{\prime}$ along each direction). Assume that

$$
\liminf _{h \rightarrow 0}<\mathcal{A}^{h}\left[v^{h}\right], v^{h}>\leq<-\chi, v>.
$$

Then $\chi+\mathcal{A}[v]=0$, and (4.41) necessarily holds with equality. 
For the sake of completeness, the proofs of Lemmas 6 and 7 are given in the Appendix, as well as the proofs of the technical Lemmas T.1 and T.2 below.

Taking advantage of the "continuous" form (3.20),(3.21) of the discrete problem $\left(\mathcal{S}^{h}\right)$, we can prove the convergence of finite volume approximate solutions in the same way, using the discrete a priori estimates shown in Propositions 1 and 3(i), using next Lemma 6 and using finally Lemma 7 together with the essential consistency result of Theorem 3.

Proof of TheOREM 2: Let $\bar{v}^{h}$ be the solution of $\left(\mathcal{S}^{h}\right)$. Let $v^{h}$ be a corresponding interpolated solution, and let $\mathcal{A}^{h}$ be the finite volume approximate of the operator $\mathcal{A}$ in (1.1) (cf. (3.28),(3.29)). Note that all the convergences we state below take place up to exstraction of a subsequence.

(A) By Proposition 1(i), $\left\|\mathcal{D}_{\perp}^{h}\left[\bar{v}^{h}\right]\right\|_{L^{p}(Q)} \leq$ const uniformly in $h$ so that the family $\left(v^{h}\right)_{h}$ is bounded in $E$, by (2.19). Hence there exists a function $v \in E$ such that $v^{h} \rightarrow v$ in $E$ as $h \rightarrow 0$. By (2.17), one also has $\bar{v}^{h} \rightarrow v$ in $L^{p}(Q)$.

(B) We claim that the family $\left(u^{h}\right)_{h}$ given by (3.22) is relatively compact in $L^{1}(Q)$. Indeed, let us check the assumptions of Lemma 6. We have $u^{h}{ }_{t}=\operatorname{div} a_{p}\left(\mathcal{G}^{h}\left[v^{h}\right]\right)$ in $\mathcal{D}^{\prime}(Q)$ by (3.20),(3.21), and the family $\left(a_{p}\left(\mathcal{G}^{h}\left[v^{h}\right]\right)\right)_{h}$ is bounded in $L^{p^{\prime}}(Q)$ by Proposition 1(i), (2.19) and Proposition 3(i) (note that $\left(\mathcal{A}^{h}\left[v^{h}\right]\right)_{h}$ is thus bounded in $\left.E^{\prime}\right)$. Furthermore, (3.22) yields

$$
\left\|u^{h}\right\|_{L^{1}(Q)} \leq 2 \iint_{Q}\left|b\left(\bar{v}^{h}\right)\right|+k^{h} \sum_{K} \mathfrak{m}(K)\left|u_{K}^{0}\right|
$$

and one has

$$
|b(z)| \leq \delta B(z)+\sup _{|\zeta| \leq 1 / \delta}|b(\zeta)|
$$

for all $\delta>0$ (cf., e.g., [1]). By Proposition 1(ii) and since $u_{0}^{h}=\sum_{K} \mathfrak{m}(K)\left|u_{K}^{0}\right| \rightarrow u_{0}$ in $L^{1}(\Omega)$ as $h \rightarrow 0$, it follows that $\left(u^{h}\right)_{h}$ is bounded in $L^{1}(Q)$. Finally, using Lemma 1 (ii), we can prove the following result.

Lemma T.1 Let $\left(\bar{v}^{h}\right)_{h}$ be discrete solutions to a family of problems $\left(\mathcal{S}^{h}\right)_{h}$ satisfying the assumptions of Proposition 1. Let $\left(u^{h}\right)_{h}$ be defined by (3.22). Then (4.40) holds with $\omega(\cdot)$ independent of $h$.

By Proposition 1, we can apply Lemma T.1 and then Lemma 6. Thus, there exists a function $u \in L^{1}(Q)$ such that $u^{h} \rightarrow u$ in $L^{1}(Q)$ and a.e. on $Q$. In addition, due to the monotonicity of $b(\cdot)$, we can identify $u$ with $b(v)$ :

Lemma T.2 Let $\left(\bar{v}^{h}\right)_{h}$ be discrete solutions to $\left(\mathcal{S}^{h}\right)_{h},\left(v^{h}\right)_{h}$ be corresponding interpolated solutions, and $\left(u^{h}\right)_{h}$ be defined by (3.22). If $v^{h} \rightarrow v$ in $E$ and $u^{h} \rightarrow u$ in $L^{1}(Q)$ as $h \rightarrow 0$, then $u=b(v)$.

(C) By $(\mathrm{A})$, we have $v^{h} \rightarrow v$ in $E$. We claim that $v$ is a weak solution of (1.1).

By Proposition 2, $\chi^{h}+\mathcal{A}^{h}\left[v^{h}\right]=0$ in $E^{\prime}$ and the initial condition (3.21) is verified for all $h$. The family $\left(\mathcal{A}^{h}\left[v^{h}\right]\right)_{h}$ is bounded in $E^{\prime}$ (cf. (B)), thus $\left(\chi^{h}\right)_{h}$ is weak-* relatively compact in $E^{\prime}$. By (3.23), (B), and Definition 1, we also have $\chi^{h}=u^{h}{ }_{t} \rightarrow b(v)_{t}=\chi$ in $\mathcal{D}^{\prime}(Q)$. Hence $\mathcal{A}^{h}\left[v^{h}\right]=-\chi^{h} \stackrel{*}{\rightarrow}-\chi$ in $E^{\prime}$.

Moreover, passing to the limit in (3.21), using (B) and the convergence of $u_{0}^{h}$ to $u_{0}$ in $L^{1}(\Omega)$, we get (1.5). Consequently, by the chain rule argument [1, Lemma 1.5] we have

$$
<-\chi, v>_{E^{\prime}, E}=-\int_{\Omega} \Psi(b(v(T, \cdot)))+\int_{\Omega} \Psi\left(u^{0}\right) .
$$


On the other hand, by (3.23),(3.22),(2.18), and the monotonicity of $b(\cdot)$, we have

$$
\begin{aligned}
<-\chi^{h}, v^{h}>_{E^{\prime}, E} & =-\frac{1}{k} \sum_{K, n}\left(b\left(v_{K}^{n}\right)-b\left(v_{K}^{n-1}\right)\right) \iint_{Q_{K}^{n}} v^{h}=-\sum_{K, n} \mathfrak{m}(K)\left(b\left(v_{K}^{n}\right)-b\left(v_{K}^{n-1}\right)\right) v_{K}^{n} \leq \\
& \leq-\sum_{K} \mathfrak{m}(K) \Psi\left(b\left(v_{K}^{\left[T / k^{h}\right]+1}\right)\right)+\sum_{K} \mathfrak{m}(K) \Psi\left(u_{K}^{0}\right)=-\int_{\Omega} \Psi\left(b\left(\bar{v}^{h}(T, \cdot)\right)\right)+\int_{\Omega} \Psi\left(u_{0}^{h}\right) .
\end{aligned}
$$

Recall that $\Psi\left(u_{0}^{h}\right) \rightarrow \Psi\left(u_{0}\right)$ in $L^{1}(\Omega)$. Without loss of generality, we can assume that $\bar{v}^{h}(T, \cdot) \rightarrow v(T, \cdot)$ a.e. on $\Omega$; hence by the Fatou lemma and (4.43) we get (4.41).

Next, the operators $\mathcal{A}^{h}$ are monotone. Indeed, take $\varphi \in E$ and $\left(\varphi_{K}^{n}\right)_{K, n}=\mathcal{M}^{h}[\varphi]$. Arguing as in the proof of Proposition 2, integrating by parts in $Q_{K}^{n}$, and cancelling the boundary terms, we get

$$
\begin{aligned}
<\mathcal{A}^{h}[\eta], \varphi & >_{E^{\prime}, E}=\iint_{Q} a_{p}\left(\mathcal{G}^{h}[\eta]\right) \cdot D \varphi=-\sum_{K, n} \iint_{Q_{K}^{n}} \varphi \operatorname{div} a_{p}\left(\mathcal{G}^{h}\left[v^{h}\right]\right)=-\sum_{K, n} \iint_{Q_{K}^{n}} \varphi \times \\
\times & \frac{1}{\mathfrak{m}(K)} \sum_{L \in \mathcal{N}_{K}} \int_{K \mid L} a_{p}\left(D_{K \mid L}^{n}(x)\right) d x \cdot \nu_{K, L}=-k \sum_{K, n} \varphi_{K}^{n} \sum_{L \in \mathcal{N}_{K}} \int_{K L} a_{p}\left(D_{K \mid L}^{n}(x)\right) d x \cdot \nu_{K, L} .
\end{aligned}
$$

Substituting (4.44) and applying Remark 1, we infer by property (4ii) of Definition 4 that

$$
\begin{aligned}
<\mathcal{A}^{h}[\eta] & -\mathcal{A}^{h}[\widetilde{\eta}], \eta-\widetilde{\eta}>_{E^{\prime}, E}= \\
& =\frac{1}{d} k \sum_{K L, n}\left(\left(\eta_{L}^{n}-\eta_{K}^{n}\right)-\left(\widetilde{\eta}_{L}^{n}-\widetilde{\eta}_{K}^{n}\right)\right) \int_{K L L}\left(a_{p}\left(D_{K L}^{n}(x)\right)-a_{p}\left(\widetilde{D}_{K L}^{n}(x)\right)\right) d x \cdot \nu_{K, L} \geq 0,
\end{aligned}
$$

where $\left(\eta_{K}^{n}\right)_{K, n}=\mathcal{M}^{h}[\eta],\left(D_{K L L}^{n}\right)_{K L, n}=\mathcal{D}^{h}[\eta]$, and the same for $\tilde{\eta}$.

Finally, by Theorem $3, \mathcal{A}^{h}$ converge pointwise to the hemicontinuous operator $\mathcal{A} \cdot=-\operatorname{div} a_{p}(D \cdot)$. By Lemma 7 we conclude that $\chi+\mathcal{A}[v]=0$ in $E^{\prime}$. Thus (1.4) holds and $v$ is a weak solution of (1.1).

Note that in practice, due to (3.34), property (4ii) of Definition 4 can be satisfied with an inequality which allows to minorate $\left\|\mathcal{D}_{\perp}^{h}\left[\left(v_{K}^{n}-\widetilde{v}_{K}^{n}\right)_{K, n}\right]\right\|_{L^{p}(Q)}$. In particular, this is true for the discrete gradients proposed in Section 5. In this case we can improve the convergence result.

Corollary 1 Let us require instead of (4ii) that

(4ii') for all $R>0$ there exists a function $\Xi_{R}(\cdot)$ such that $\lim _{\xi \rightarrow 0} \Xi_{R}(\xi)=0$ and for all $h$,

$$
\begin{aligned}
& \Xi_{R}\left(\frac{1}{d} k^{h} \sum_{K L, n}\left(\left(v_{L}^{n}-v_{K}^{n}\right)-\left(\widetilde{v}_{L}^{n}-\widetilde{v}_{K}^{n}\right)\right) \int_{K \mid L}\left(a_{p}\left(D_{K L}^{n}(x)\right)-a_{p}\left(\widetilde{D}_{K L L}^{n}(x)\right)\right) d x \cdot \nu_{K, L}\right) \geq \\
& \geq\left\|\mathcal{D}_{\perp}^{h}\left[\left(v_{K}^{n}-\widetilde{v}_{K}^{n}\right)_{K, n}\right]\right\|_{L^{p}(Q)} \quad \text { whenever }\left\|\mathcal{D}_{\perp}^{h}\left[\left(v_{K}^{n}\right)_{K, n}\right]\right\|_{L^{p}(Q)} \leq R,\left\|\mathcal{D}_{\perp}^{h}\left[\left(\widetilde{v}_{K}^{n}\right)_{K, n}\right]\right\|_{L^{p}(Q)} \leq R .
\end{aligned}
$$

Then $\bar{v}^{h} \rightarrow v$ strongly in $L^{p}(Q)$ under the assumptions of Theorem 2.

Proof: By (4 ii '), Proposition 1(i) and (4.45), we have

$$
\left\|\mathcal{D}_{\perp}^{h}\left[v^{h}-v\right]\right\|_{L^{p}(Q)} \leq \Xi_{C}\left(<\mathcal{A}^{h}\left[v^{h}\right]-\mathcal{A}^{h}[v], v^{h}-v>_{E^{\prime}, E}\right)
$$

for some $C>0$ and for all $h$. Coming back to (C) in the proof of Theorem 2, thanks to Lemma 7 , Theorem 3 and since $v^{h} \rightarrow v$ in $E$, we find that $\liminf _{h \rightarrow 0}<\mathcal{A}^{h}\left[v^{h}\right]-\mathcal{A}^{h}[v], v^{h}-v>_{E^{\prime}, E}=0$. Hence $\left\|\mathcal{D}_{\perp}^{h}\left[\bar{v}^{h}-v\right]\right\|_{L^{p}(Q)}=\left\|\mathcal{D}_{\perp}^{h}\left[v^{h}-v\right]\right\|_{L^{p}(Q)} \rightarrow 0$ as $h \rightarrow 0$. Since $\sum_{K, n}\left(\frac{1}{k^{h} \mathfrak{m}(K)} \iint_{Q_{K}^{n}} v\right) \mathbb{1}_{Q_{K}^{n}} \rightarrow v$ in $L^{p}(Q)$ and by Lemma $1(\mathrm{i})$, we have $\left\|\bar{v}^{h}-v\right\|_{L^{p}(Q)} \rightarrow 0$ as $h \rightarrow 0$. 


\section{EXAMPLES OF ADMISSIBLE METHODS}

By an admissible method, we mean a method which provides an admissible gradient approximation and weakly proportional meshes satisfying the interpolation property. Recall that in this case, we have the convergence of the finite volume approximation (cf. Theorem 2). In this section, we prove that such admissible methods exist.

\subsection{ON DISCRETE GRADIENTS}

In this section we construct an admissible gradient for a family $\left(\mathcal{T}^{h}\right)_{h}$ of finite volume meshes of the Voronoï kind dual to a family $\left(\widehat{\mathcal{T}}^{h}\right)_{h}$ of triangular meshes.

Let us introduce some notation. We use $\widehat{O}$ to denote a triangle of the mesh $\widehat{\mathcal{T}}^{h}$; for all $\hat{O} \in \widehat{\mathcal{T}}^{h}$, there exist $K, L, M \in \mathcal{T}^{h}$ such that $\hat{O}=\Delta x_{K} x_{L} x_{M}$ (the triangle with the corners $x_{K}, x_{L}, x_{M}$ ). The three interfaces $K L, L M, M K$ intersect at point $x_{\hat{O}}$, which is the center of the circumscribed circle of triangle $\hat{o}$. We require it to be inside $\hat{O}$. Let us denote by $S_{\hat{O}}, S_{K, L}, S_{L, M}, S_{M, K}$ the surfaces of $\Delta x_{K} x_{L} x_{M}$, $\Delta x_{\hat{O}} x_{K} x_{L}, \Delta x_{\hat{O}} x_{L} x_{M}, \Delta x_{\hat{O}} x_{M} x_{K}$, respectively. One has $S_{\hat{O}}=S_{K, L}+S_{L, M}+S_{M, K}$. Recall that $\nu_{K, L}=\overrightarrow{x_{K} x_{L}} / d_{K, L}, \nu_{L, M}=\overrightarrow{x_{L} x_{M}} / d_{L, M}, \nu_{M, K}=\overrightarrow{x_{M} x_{K}} / d_{M, K}$. Note the following elementary lemma.

Lemma 8 Let $\hat{O}=\Delta x_{K} x_{L} x_{M}$ be a triangle in $\mathbb{R}^{2}$, let $x_{\hat{O}}$ be the center of its circumscribed circle, and let $x_{\hat{O}} \in \Delta x_{K} x_{L} x_{M}$. With the above notation, for all $r$ in $\mathbb{R}^{2}$, we have

$$
r=\frac{2}{S_{\widehat{O}}}\left\{S_{K, L}\left(r \cdot \nu_{K, L}\right) \nu_{K, L}+S_{L, M}\left(r \cdot \nu_{L, M}\right) \nu_{L, M}+S_{M, K}\left(r \cdot \nu_{M, K}\right) \nu_{M, K}\right\} .
$$

This property can be generalized to any polygon in $\mathbb{R}^{2}$ which admits the circumscribed circle.

Further, for $\hat{O} \in \widehat{\mathcal{T}}^{h}$ such that $\hat{O}=\Delta x_{K} x_{L} x_{M}$, let $v^{h, 0}{ }_{\hat{O}}: \mathbb{R}^{2} \rightarrow \mathbb{R}^{N}$ be the affine function that takes the values $v_{K}, v_{L}, v_{M}$ at the points $x_{K}, x_{L}, x_{M}$, respectively. The discrete gradient operator $\mathcal{D}^{h, 0}=$ $\mathcal{L}^{h} \circ \mathcal{D}^{h, 0}$ is defined by

$$
\mathcal{D}^{h, 0}:\left(v_{K}\right)_{K} \mapsto \sum_{\hat{o} \in \widehat{\mathcal{T}}^{h}} D v^{h, 0} \hat{o}(x) \mathbb{1}_{\hat{O}}(x) .
$$

In the case of structured hexagonal meshes, as well as that of structured rectangular ones, the family $\left(\mathcal{D}^{h, 0}\right)_{h}$ is admissible (this will be proved in Proposition 4, as a particular case). In general, this construction does not work. Indeed, if the points $x_{K}$ are not the barycenters of $K \in \mathcal{T}^{h}$, property (4 iv) fails.

This can be overcome, for instance, in the following way. For all $K \in \mathcal{T}^{h}$, let $y_{K}$ be the barycenter of $K$ and set $\sigma_{K}=x_{K}-y_{K}$. For $\hat{O} \in \widehat{\mathcal{T}}^{h}$ such that $\hat{O}=\Delta x_{K} x_{L} x_{M}$, let $v_{\hat{O}}^{h}: \mathbb{R}^{2} \rightarrow \mathbb{R}^{N}$ be the affine function that takes the values $v_{K}, v_{L}, v_{M}$ at the points $y_{K}, y_{L}, y_{M}$, respectively. The discrete gradient operator $\mathcal{D}^{h}=\mathcal{L}^{h} \circ \mathcal{D}^{h}$ is defined by

$$
\mathcal{D}^{h}:\left(v_{K}\right)_{K} \mapsto \sum_{\hat{o} \in \widehat{\mathcal{T}}^{h}} D v_{\widehat{O}}^{h}(x) \mathbb{1}_{\widehat{o}}(x)
$$

i.e., the affine interpolation over the triangle $\Delta y_{K} y_{L} y_{M}$ is actually used in the triangle $\Delta x_{K} x_{L} x_{M}$.

We will take advantage of considering $\mathcal{D}^{h}$ as a perturbation of $\mathcal{D}^{h, 0}$. For all $\widehat{O} \in \widehat{\mathcal{T}}^{h}$, let us define the correction operators

$R_{\widehat{O}}: r \in \mathbb{R}^{2} \mapsto \frac{2}{S_{\widehat{O}}}\left\{S_{K, L}\left(r \cdot \frac{\sigma_{L}-\sigma_{K}}{d_{K, L}}\right) \nu_{K, L}+S_{L, M}\left(r \cdot \frac{\sigma_{M}-\sigma_{L}}{d_{L, M}}\right) \nu_{L, M}+S_{M, K}\left(r \cdot \frac{\sigma_{K}-\sigma_{M}}{d_{M, K}}\right) \nu_{M, K}\right\}$, 
with the notation introduced above. We need to guarantee that the Euclidiean norm of $R_{\hat{O}}$ is less than $\min \{p-1,1 /(p-1)\}$ for all $\hat{o} \in \mathcal{T}^{h}$.

Proposition 4 Assume that $\left(\mathcal{T}^{h}\right)_{h}$ is a family of meshes dual to a family of meshes $\left(\widehat{\mathcal{T}}^{h}\right)_{h}$ such that all $\hat{o} \in \widehat{\mathcal{T}}^{h}$ are triangles with angles less than or equal to $\pi / 2$. Assume that for all $h$, for all $\hat{o} \in \widehat{\mathcal{T}}^{h}$,

$$
\frac{2}{S_{\widehat{O}}}\left\{S_{K, L} \frac{\left|\sigma_{L}-\sigma_{K}\right|}{d_{K, L}}+S_{L, M} \frac{\left|\sigma_{M}-\sigma_{L}\right|}{d_{L, M}}+S_{M, K} \frac{\left|\sigma_{K}-\sigma_{M}\right|}{d_{M, K}}\right\}<\min \{p-1,1 /(p-1)\},
$$

where $\sigma_{K}$ is the difference between the "center" $x_{K}$ of the volume $K$ and its barycenter, etc.

Then the family of discrete gradient operators $\left(\mathcal{D}^{h}\right)_{h}$ on $\left(\mathcal{T}^{h}\right)_{h}$ defined by (5.46) is admissible in the sense of Definition 4.

Proof : Since, for any affine function $w$ on $K$, one has $\frac{1}{\mathfrak{m}(K)} \int_{K} w(x) d x=w\left(y_{K}\right)$, where $y_{K}$ is the barycenter of $K$, property ( 4 iv) holds for $\mathcal{D}^{h}$ (with $\varsigma=1$, by construction). Next, (4i) is clear.

Let us establish the relation between $\mathcal{D}^{h, 0}$ and $\mathcal{D}^{h}$. Denote by $D_{\hat{O}}^{0}, D_{\widehat{O}}$ the values on $\hat{O}$ of $\mathcal{D}^{h, 0}\left[\left(v_{K}\right)_{K}\right]$ and $\mathcal{D}^{h}\left[\left(v_{K}\right)_{K}\right]$, respectively. Let us show that for all $\hat{O} \in \widehat{\mathcal{T}}^{h}$,

$$
D_{\widehat{O}}^{0}=\left(I-R_{\widehat{O}}\right) D_{\hat{o}} .
$$

Indeed, if $\hat{O}=\Delta x_{K} x_{L} x_{M}$, one has

$$
\begin{aligned}
D_{\hat{O}} \cdot \nu_{K, L} & =\frac{v_{\hat{O}}^{h}\left(x_{L}\right)-v_{\hat{O}}^{h}\left(x_{K}\right)}{d_{K, L}}=\frac{\left(v_{L}+D_{\hat{O}} \cdot \sigma_{L}\right)-\left(v_{K}+D_{\hat{O}} \cdot \sigma_{K}\right)}{d_{K, L}}= \\
& =\frac{v_{L}-v_{K}}{d_{K, L}}+D_{\hat{O}} \cdot \frac{\sigma_{L}-\sigma_{K}}{d_{K, L}}=D_{\hat{O}}^{0} \cdot \nu_{K, L}+D_{\hat{O}} \cdot \frac{\sigma_{L}-\sigma_{K}}{d_{K, L}} .
\end{aligned}
$$

Writing the same relation for $L, M$ and $M, K$, from Lemma 8, we get $D_{\widehat{O}}-D_{\widehat{O}}^{0}=R_{\widehat{O}} D_{\widehat{O}}$, whence (5.47) follows.

By Lemma 8 and the definition of $\mathcal{D}_{\perp}^{h}=\mathcal{L}^{h} \circ \mathcal{D}_{\perp}^{h}$ for all $\hat{o} \in \widehat{\mathcal{T}}^{h}$ such that $\hat{O}=\Delta x_{K} x_{L} x_{M}$ we have

$$
\begin{aligned}
& \int_{\widehat{O}}\left|\mathcal{D}^{h, 0}\left[\left(v_{K}\right)_{K}\right]\right|^{p}=S_{\widehat{O}}\left|\mathcal{D}^{h, 0}\left[\left(v_{K}\right)_{K}\right]\right|^{p} \leq \\
& \quad \leq C^{*}\left\{S_{K, L}\left|\frac{v_{L}-v_{K}}{d_{K, L}}\right|^{p}+S_{L, M}\left|\frac{v_{M}-v_{L}}{d_{L, M}}\right|^{p}+S_{M, K}\left|\frac{v_{K}-v_{M}}{d_{M, K}}\right|^{p}\right\}=C^{*} \int_{\widehat{O}}\left|\mathcal{D}_{\perp}^{h}\left[\left(v_{K}\right)_{K}\right]\right|^{p}
\end{aligned}
$$

with a constant $C^{*}$ that depends only on $p$. Since for given $\widetilde{K} \in \mathcal{T}^{h}$ and $\widehat{O} \in \widehat{\mathcal{T}}^{h}$ we have $\widetilde{K} \cap \widehat{O} \neq \varnothing$ if and only if $\hat{O} \in \Upsilon_{1}(\widetilde{K})$, it follows that property $(4 \mathrm{v})$ holds for the discrete gradient $\mathcal{D}^{h, 0}$, with $\varsigma=1$. Now set $\theta_{\hat{O}}=\left\|R_{\widehat{O}}\right\|$. We have $\theta_{\widehat{O}}<1$. One has $\left|D_{\widehat{O}}\right| \leq\left\|\left(I-R_{\widehat{O}}\right)^{-1}\right\|\left|D_{\hat{O}}^{0}\right| \leq \frac{1}{1-\theta_{\hat{O}}}\left|D_{\widehat{O}}^{0}\right| ;$ therefore $(4 \mathrm{v})$ also holds for $\mathcal{D}^{h}$.

Next, each term in the sum in (4iii) splits into two terms corresponding to the two parts of the interface $K L$ included in different triangles $\widehat{O}_{1}, \widehat{O}_{2} \in \widehat{\mathcal{T}}^{h}$. Let us write down all the terms corresponding to the same triangle $\widehat{O} \in \widehat{\mathcal{T}}^{h}, \widehat{O}=\Delta x_{K} x_{L} x_{M}$, combine them using Lemma 8, and estimate using (5.47):

$$
\begin{aligned}
& S_{K, L}\left(a_{p}\left(D_{\hat{O}}\right) \cdot \nu_{K, L}\right)\left(D_{\widehat{O}}^{0} \cdot \nu_{K, L}\right)+S_{L, M}\left(a_{p}\left(D_{\widehat{O}}\right) \cdot \nu_{L, M}\right)\left(D_{\widehat{O}}^{0} \cdot \nu_{L, M}\right)+ \\
& +S_{M, K}\left(a_{p}\left(D_{\widehat{O}}\right) \cdot \nu_{M, K}\right)\left(D_{\widehat{O}}^{0} \cdot \nu_{M, K}\right)=\frac{S_{\widehat{O}}}{2} a_{p}\left(D_{\hat{O}}\right) \cdot D_{\widehat{O}}^{0}=\frac{S_{\widehat{O}}}{2} a_{p}\left(D_{\widehat{O}}\right) \cdot\left(I-R_{\widehat{O}}\right) D_{\widehat{O}} \geq \\
& \geq \frac{1-\theta_{\hat{O}}}{2} S_{\widehat{O}}\left|D_{\widehat{O}}\right|^{p} \geq \frac{1-\theta_{\hat{O}}}{2\left(1+\theta_{\hat{O}}\right)^{p}} S_{\widehat{O}}\left|D_{\widehat{O}}^{0}\right|^{p} \text {. }
\end{aligned}
$$


Property (4iii) for $\mathcal{D}^{h}$ follows, because one has $\left|D^{0}\right| \geq\left|D_{\widehat{O}}^{0} \cdot \nu_{K, L}\right|=\frac{\left|v_{L}-v_{K}\right|}{d_{K, L}}=D_{\perp, K L}^{h}$, so that

$$
\sum_{\hat{O} \in \widehat{\mathcal{T}}^{h}} S_{\widehat{O}}\left|D_{\widehat{O}}^{0}\right|^{p}=\left\|\mathcal{D}^{h, 0}\left[\left(v_{K}\right)_{K}\right]\right\|_{L^{p}(\Omega)}^{p} \geq\left\|\mathcal{D}_{\perp}^{h}\left[\left(v_{K}\right)\right]\right\|_{L^{p}(\Omega)}^{p}
$$

The proof of (4ii) is similar. Denoting the values $\widetilde{D}_{\widehat{O}}^{0}, \widetilde{D}_{\hat{O}}$ of $\mathcal{D}^{h, 0}\left[\left(\widetilde{v}_{K}\right)_{K}\right]$ and $\mathcal{D}^{h}\left[\left(\widetilde{v}_{K}\right)_{K}\right]$, respectively, on $\hat{O}$, one can rewrite the sum in (4ii) as

$$
\begin{aligned}
& \sum_{\hat{o} \in \widehat{\mathcal{T}}^{h}}\left\{S_{K, L}\left(\left(a_{p}\left(D_{\hat{O}}\right)-a_{p}\left(\widetilde{D}_{\hat{O}}\right)\right) \cdot \nu_{K, L}\right)\left(\left(D_{\hat{O}}^{0}-\widetilde{D}_{\hat{O}}^{0}\right) \cdot \nu_{K, L}\right)+\right. \\
& +S_{L, M}\left(\left(a_{p}\left(D_{\widehat{O}}\right)-a_{p}\left(\widetilde{D}_{\hat{O}}\right)\right) \cdot \nu_{L, M}\right)\left(\left(D_{\widehat{O}}^{0}-\widetilde{D}_{\hat{o}}^{0}\right) \cdot \nu_{L, M}\right)+ \\
& \left.+S_{M, K}\left(\left(a_{p}\left(D_{\widehat{O}}\right)-a_{p}\left(\widetilde{D}_{\widehat{O}}\right)\right) \cdot \nu_{M, K}\right)\left(\left(D_{\widehat{O}}^{0}-\widetilde{D}_{\widehat{O}}^{0}\right) \cdot \nu_{M, K}\right)\right\} \\
& =\frac{1}{2} \sum_{\hat{O} \in \widehat{\mathcal{T}}^{h}} S_{\widehat{O}}\left(a_{p}\left(D_{\hat{O}}\right)-a_{p}\left(\widetilde{D}_{\widehat{O}}\right)\right) \cdot\left(D_{\widehat{O}}^{0}-\widetilde{D}_{\widehat{O}}^{0}\right) .
\end{aligned}
$$

Using (5.47) and denoting by $H$ the Hessian matrix of the function $x \in \mathbb{R}^{2} \mapsto \frac{1}{p}|x|^{p}$, we get

$$
\left(a_{p}\left(D_{\hat{o}}\right)-a_{p}\left(\widetilde{D}_{\hat{o}}\right)\right) \cdot\left(D_{\widehat{o}}^{0}-\widetilde{D}_{\hat{o}}^{0}\right)=\left(D_{\hat{o}}-\widetilde{D}_{\hat{o}}\right)^{t}\left[\int_{0}^{1} H\left(\widetilde{D}_{\hat{o}}+\tau\left(D_{\widehat{o}}-\widetilde{D}_{\hat{o}}\right)\right) d \tau\left(I-R_{\widehat{O}}\right)\right]\left(D_{\widehat{o}}-\widetilde{D}_{\hat{o}}\right) .
$$

For all $x \in \mathbb{R}^{2}, x \neq 0, H(x)$ is a symmetric matrix with positive eigenvalues $\lambda_{1}, \lambda_{2}$ such that $\lambda_{1} / \lambda_{2}=$ $p-1$. Thus the condition $\left\|R_{\hat{O}}\right\|<\min \{p-1,1 /(p-1)\}$ ensures that, for all $\tau \in[0,1]$,

$$
r^{t}\left[H\left(\widetilde{D}_{\hat{O}}+\tau\left(D_{\widehat{O}}-\widetilde{D}_{\widehat{O}}\right)\right)\left(I-R_{\widehat{O}}\right)\right] r \geq a r^{t}\left[H\left(\widetilde{D}_{\widehat{O}}+\tau\left(D_{\widehat{O}}-\widetilde{D}_{\hat{O}}\right)\right)\right] r>0
$$

for all $r \in \mathbb{R}^{2}, r \neq 0$, with some constant $a>0$. Now (4ii) follows. Moreover, ( 4 ii ') also follows from (5.49),(5.50), due to the inequalities (3.34). For $p \geq 2$, one can take $\Xi_{R}(\xi)=$ const $\xi^{1 / p}$ in (4ii'), and for $1<p \leq 2$, using the inverse Hölder inequality, one can take $\Xi_{R}(\xi)=$ const $\left(\xi R^{2-p}\right)^{1 / 2}$.

Remark 3 In the three-dimensional case, for a family of meshes dual to a family of structured tetrahedrical meshes, the gradient $\mathcal{D}^{h, 0}$ constructed in the same way as above is admissible. The proof is the same, using an easy symmetry observation instead of Lemma 8.

\subsection{ON INTERPOLATED SOLUTIONS}

First note that it is sufficient to prove the following interpolation property of $\left(\mathcal{T}^{h}\right)_{h}$ in $W_{0}^{1, p}(\Omega)$ :

Definition $5^{\prime} \quad A$ family of grids $\left(\mathcal{T}^{h}\right)_{h}$ has the interpolation property in $W_{0}^{1, p}(\Omega)$ if, for any family $\left(\bar{v}^{h}\right)_{h}$ of functions such that $\left.\bar{v}^{h}\right|_{K}=v_{K} \equiv$ const for each $K \in \mathcal{T}^{h}$, with $v_{K}=0$ for $K \in \mathcal{T}_{\text {ext }}^{h}$ and with $\left\|\mathcal{D}_{\perp}^{h}\left[\bar{v}^{h}\right]\right\|_{L^{p}(\Omega)} \leq C$ for all $h$, there exists a family $\left(v^{h}\right)_{h} \subset W_{0}^{1, p}(\Omega)$ such that

$$
\begin{gathered}
\left\|v^{h}-\bar{v}^{h}\right\|_{L^{p}(\Omega)} \rightarrow 0 \quad \text { as } h \rightarrow 0, \\
\frac{1}{\mathfrak{m}(K)} \int_{K} v^{h}=v_{K} \quad \text { for all } K \in \mathcal{T}^{h}, \\
\left\|v^{h}\right\|_{W_{0}^{1, p}(\Omega)} \leq c \times\left\|\mathcal{D}_{\perp}^{h}\left[\bar{v}^{h}\right]\right\|_{L^{p}(\Omega)} \quad \text { with a constant } c \text { independent of } h .
\end{gathered}
$$


Indeed, we obtain the interpolation property in $E$ with the function $I: C \mapsto c \times C$ by taking $v^{h}$ constant on each $I^{n}$ and summing in $n \in\left\{1, \ldots,\left[T / k^{h}\right]+1\right\}$.

Lemma 9 Let $\left(\mathcal{T}^{h}\right)_{h}$ be a strongly proportional family of finite volume meshes of $\Omega \subset \mathbb{R}^{d}$. Then it has the interpolation property in $W_{0}^{1, p}(\Omega)$.

Proof : Take discrete solutions $\bar{v}^{h}=\sum_{K} v_{K} \mathbb{1}_{K}$ on each of $\mathcal{T}^{h}$ such that $\left\|\mathcal{D}_{\perp}^{h}\left[\bar{v}^{h}\right]\right\|_{L^{p}(Q)} \leq C$ for all $h$. Let $\widetilde{C}$ denote a generic constant depending only on $p, d$ and $\Omega$. We first convolute $\bar{v}^{h}$ with a standard mollifier, and then restore the average over each mesh volume. More exactly, let $\zeta_{*}$ be the strong proportionality constant in (2.7); set $\nu=h \zeta_{*} / 2$ and

$$
v^{h,-1}=\int_{\mathbb{R}^{d}} \rho_{h}(y) \bar{v}^{h}(x-y) d y,
$$

where $\operatorname{supp} \rho_{h} \subset\left\{y \in \mathbb{R}^{d}|| y \mid<\nu\right\}, \rho_{h} \geq 0$ and $\int_{\mathbb{R}^{d}} \rho_{h}(y) d y=1$. Note that we have $\| v^{h,-1}-$ $\bar{v}^{h} \|_{L^{p}(\Omega)} \rightarrow 0$ as $h \rightarrow 0$ (cf., e.g., [8, Th.IV.22]). Moreover, using [8, Prop.IX.3], by (5.51) and the Hölder inequality we get

$$
\begin{aligned}
& \left\|D v^{h,-1}\right\|_{L^{p}(\Omega)}^{p}=\limsup _{\Delta x \rightarrow 0} \frac{1}{\mid \Delta x p^{p}} \int_{\Omega}\left|v^{h,-1}(x+\Delta x)-v^{h,-1}(x)\right|^{p} d x \leq \\
& \leq \limsup _{\Delta x \rightarrow 0} \frac{1}{|\Delta x|^{p}} \int_{\Omega}\left(\int_{\mathbb{R}^{d}} \rho_{h}(y) d y\right)^{p-1} \int_{\mathbb{R}^{d}} \rho_{h}(y)\left|\bar{v}^{h}(x-y+\Delta x)-\bar{v}^{h}(x-y)\right|^{p} d y d x \leq \\
& \leq \limsup _{\Delta x \rightarrow 0} \frac{1}{|\Delta x|^{p}} \int_{\mathbb{R}^{d}} \rho_{h}(y) \int_{\mathbb{R}^{d}}\left|\bar{v}^{h}(z+\Delta x)-\bar{v}^{h}(z)\right|^{p} d z d y=\limsup _{\Delta x \rightarrow 0} \frac{1}{|\Delta x|^{p}} \int_{\mathbb{R}^{d}}\left|\bar{v}^{h}(z+\Delta x)-\bar{v}^{h}(z)\right|^{p} d z .
\end{aligned}
$$

Hence, by Lemma 1 (iii), we find, choosing $\Delta=h$

$$
\left\|D v^{h,-1}\right\|_{L^{p}(\Omega)}^{p} \leq \limsup _{\Delta \rightarrow 0} \frac{1}{\Delta^{p}} \Delta(\Delta+2 h)^{p-1} \times \widetilde{C}\left\|\mathcal{D}_{\perp}^{h}\left[\bar{v}^{h}\right]\right\|_{L^{p}(\Omega)}^{p} \leq \widetilde{C} \times C^{p} .
$$

Next, set $\Omega_{-\nu}=\{x \in \Omega \mid \operatorname{dist}(x, \partial \Omega) \geq \nu\}$ and

$$
v^{h, 0}(x)= \begin{cases}0, & x \notin \Omega \\ v^{h,-1}(x) \frac{\operatorname{dist}\left(x, \Omega_{-\nu}\right)}{\nu}, & x \in \Omega .\end{cases}
$$

We have $v^{h, 0} \in W_{0}^{1, p}(\Omega)$; moreover, $\left|D v^{h, 0}\right| \leq\left|D v^{h,-1}\right|+\left|v^{h,-1}\right| \frac{1}{\nu} \mathbb{1}_{\Omega \backslash \Omega_{-\nu}}$. Note that, since $\Omega$ is a polygonal domain, we can split $\Omega \backslash \Omega_{-\nu}$ into a finite number of domains, each one included in a strip of thickness $\nu$. Hence by the Poincaré inequality, $\int_{\Omega_{\backslash} \backslash \Omega_{-}}\left|v^{h,-1}\right|^{p} \leq \widetilde{C} \nu^{p} \int_{\Omega_{\backslash} \Omega_{-}}\left|D v^{h,-1}\right|^{p}$. Therefore we have $\left\|v^{h, 0}\right\|_{W_{0}^{1, p}(\Omega)} \leq \widetilde{C}\left\|D v^{h,-1}\right\|_{L^{p}(\Omega)} \leq \widetilde{C} \times C$. Moreover,

$$
\left\|v^{h, 0}-v^{h,-1}\right\|_{L^{p}(\Omega)}^{p} \leq \int_{\Omega \backslash \Omega_{-\nu}}\left|v^{h,-1}\right|^{p} \leq \widetilde{C}\left(h \zeta_{*}\right)^{p}\left\|D v^{h,-1}\right\|_{L^{p}(\Omega)}^{p} \rightarrow 0 \text { as } h \rightarrow 0 .
$$

Now take a continuously differentiable function $\pi: \mathbb{R}^{2} \rightarrow \mathbb{R}^{+}$such that $\operatorname{supp} \pi=\left\{x \in \mathbb{R}^{2}|| x \mid \leq 1\right\}$ and $\int_{\mathbb{R}^{d}} \pi=1$. For all $K \in \mathcal{T}_{\text {int }}^{h}$, set $\varphi_{K}=\frac{\mathfrak{m}(K)}{\left(h \zeta_{*}\right)^{d}} \pi\left(\frac{x-x_{K}}{h \zeta_{*}}\right)$ (for boundary volumes $K$ of non zero measure, i.e., if $x_{K} \in \partial \Omega$, an easy modification is needed in order to keep the trace on $\partial \Omega$ equal to zero). Set

$$
v^{h}=v^{h, 0}+\sum_{K} \alpha_{K} \varphi_{K}, \quad \text { with } \quad \alpha_{K}=v_{K}-\frac{1}{\mathfrak{m}(K)} \int_{K} v^{h, 0}
$$


Since $\operatorname{supp} \varphi_{K} \subset K$, by the choice of $\alpha_{K}$, the family $\left(v^{h}\right)_{h}$ verifies $\left(2.18^{\prime}\right)$.

Denote by $c$ a generic constant only depending on $\zeta_{*}$. Note that by the strong proportionality of $\left(\mathcal{T}^{h}\right)_{h}$, $\frac{\mathfrak{m}(K)}{h^{d}} \leq c$ for all $K \in \mathcal{T}^{h}$ for all $h$. Note that $\bar{v}^{h} \equiv v_{K}$ on $K$, so that by the construction of $v^{h,-1}, v^{h, 0}$, we have $v^{h, 0}(x)=v_{K}$ whenever $\left|x-x_{K}\right|<h \zeta_{*} / 2$. Moreover, since $\mathfrak{d}(K) \leq h$, Lemma 3(i) yields

$$
\int_{K}\left|v^{h, 0}-\bar{v}^{h}\right|^{p}=\int_{K}\left|v^{h, 0}-v_{K}\right|^{p} \leq c h^{p} \int_{K}\left|D v^{h, 0}\right|^{p} .
$$

By (5.52) and the Hölder inequality

$$
\begin{aligned}
\int_{\Omega}\left|v^{h}-v^{h, 0}\right|^{p}=\sum_{K}\left|\alpha_{K}\right|^{p} \int_{K}\left|\varphi_{K}\right|^{p} \leq c \sum_{K} \frac{1}{\mathfrak{m}(K)^{p}}\left|\int_{K} \bar{v}^{h}-\int_{K} v^{h, 0}\right|^{p} \mathfrak{m}(K)\left(\frac{\mathfrak{m}(K)}{h^{d}}\right)^{p} \leq \\
\leq c \sum_{K} \frac{1}{\mathfrak{m}(K)^{p}} \mathfrak{m}(K)^{p^{\prime} / p} \int_{K}\left|\bar{v}^{h}-v^{h, 0}\right|^{p} \mathfrak{m}(K)=c \int_{\Omega}\left|\bar{v}^{h}-v^{h, 0}\right|^{p} \rightarrow 0
\end{aligned}
$$

as $h \rightarrow 0$. Thus $\left(v^{h}\right)_{h}$ satisfies $\left(2.17^{\prime}\right)$. In the same manner, using (5.53) we have

$$
\begin{aligned}
\int_{\Omega}\left|D v^{h}-D v^{h, 0}\right|^{p} & =\sum_{K}\left|\alpha_{K}\right|^{p} \int_{K}\left|D \varphi_{K}\right|^{p} \leq \sum_{K} c \sum_{K} \frac{1}{\mathfrak{m}(K)^{p}} \mathfrak{m}(K)^{p^{\prime} / p} \int_{K}\left|\bar{v}^{h}-v^{h, 0}\right|^{p} \times \\
\times \mathfrak{m}(K)\left(\frac{\mathfrak{m}(K)}{h^{d+1}}\right)^{p} & \leq c \sum_{K} \frac{1}{h^{p}} \int_{K}\left|\bar{v}^{h}-v^{h, 0}\right|^{p} \leq c \int_{\Omega}\left|D v^{h, 0}\right|^{p} .
\end{aligned}
$$

Hence $\left\|v^{h}\right\|_{W_{0}^{1, p}(\Omega)} \leq c\left\|v^{h, 0}\right\|_{W_{0}^{1, p}(\Omega)} \leq c \times \widetilde{C} \times C$, so that $\left(v^{h}\right)_{h}$ satisfies $\left(2.19^{\prime}\right)$. Thus $\left(v^{h}\right)_{h}$ can be chosen as interpolated solutions for $\left(\bar{v}^{h}\right)_{h}$.

Remark 4 The interpolation property can fail on weakly proportional meshes, at least for $p>2$.

Indeed, consider $\Omega=(0,1)^{2}$. For $s \geq 2$, let $\mathcal{T}^{s}$ be the finite volume mesh of $\Omega$ such that $\mathcal{T}_{\text {int }}^{s}=$ $\left\{K^{s}, L^{s}\right\}$ where $K^{s}=\{(x, y) \in \Omega \mid x+y<1 / s\}$ with $x_{K^{s}}=\left(\frac{1}{4 s}, \frac{1}{4 s}\right)$, and $L^{s}$ is the interior of the complementary of $K^{s}$ with $x_{L^{s}}=\left(\frac{1}{2}, \frac{1}{2}\right)$. Take $\bar{v}^{s}$ such that $\bar{v}^{s} \equiv s^{1 / p}$ on $K^{s}$ and $\bar{v}^{s} \equiv 0$ on $L^{s}$. Then $\int_{\Omega}\left|\mathcal{D}_{\perp}^{h}\left[\bar{v}^{s}\right]\right|^{p} \leq$ const uniformly in $s$. If there exist $v^{s} \in W_{0}^{1, p}(\Omega)$ interpolated solutions for $\bar{v}^{s}$, we have $\left\|v^{s}\right\|_{W_{0}^{1, p}(\Omega)} \leq$ const. Hence by the standard embedding theorem, $v^{s}$ are uniformly bounded. This contradicts the fact that $\frac{1}{\mathfrak{m}\left(K^{s}\right)} \int_{K^{s}} v^{s}=s^{1 / p} \rightarrow+\infty$ as $s \rightarrow+\infty$.

Nevertheless, we have the following result in the situation close to that of Proposition 4.

Lemma 10 Assume that $\left(\mathcal{T}^{h}\right)_{h}$ is a weakly proportional family of meshes of $\Omega \subset \mathbb{R}^{2}$ dual to a family of meshes $\left(\widehat{\mathcal{T}}^{h}\right)_{h}$ such that all $\hat{O} \in \widehat{\mathcal{T}}^{h}$ are triangles with angles less than or equal to $\pi / 2$. Then $\left(\mathcal{T}^{h}\right)_{h}$ has the interpolation property in $W_{0}^{1, p}(\Omega)$.

Proof : Take discrete solutions $\bar{v}^{h}=\sum_{K} v_{K} \mathbb{1}_{K}$ on each of $\mathcal{T}^{h}$ such that $\left\|\mathcal{D}_{\perp}^{h}\left[\bar{v}^{h}\right]\right\|_{L^{p}(Q)} \leq C$ for all $h$. Let $v^{h, 0}$ be the continuous piecewise affine function on $\Omega$ that interpolates the values $v_{K}, v_{L}, v_{M}$ at the points $x_{K}, x_{L}, x_{M}$ over $\hat{O}$, for all $\hat{O} \in \widehat{\mathcal{T}}^{h}, \hat{O}=\Delta x_{K} x_{L} x_{M}$ (we use the construction and notation of Section 5.1). We have $v^{h, 0} \in W_{0}^{1, p}(\Omega)$ and $D v^{h, 0} \equiv \mathcal{D}^{h, 0}\left[\bar{v}^{h}\right]$, so that (5.48) yields $\left\|D v^{h, 0}\right\|_{L^{p}(\Omega)} \leq c \times C$, where $c$ denotes a generic constant that depends only on $p$ and on $\zeta^{*}$. Note that for all $x \in K \in \mathcal{T}^{h}$,

$$
\left|v^{h, 0}(x)-\bar{v}^{h}(x)\right|=\left|v^{h, 0}(x)-v^{h, 0}\left(x_{K}\right)\right| \leq \mathfrak{d}(K)\left|D v^{h, 0}(x)\right| .
$$

Hence $\left\|v^{h, 0}-\bar{v}^{h}\right\|_{L^{p}(\Omega)} \leq h\left\|D v^{h, 0}\right\|_{L^{p}(\Omega)} \rightarrow 0$ as $h \rightarrow 0$. 
Next we proceed as in Lemma 9. Using the weak proportionality of $\left(\mathcal{T}^{h}\right)_{h}$, we set $\varphi_{K}=\frac{\mathfrak{m}(K)}{\left(\zeta^{*} \mathfrak{d}(K)\right)^{2}} \pi\left(\frac{x-x_{K}}{\zeta^{*} \mathfrak{d}(K)}\right)$ for all $K \in \mathcal{T}_{\text {int }}^{h}$ and we define $v^{h}$ by (5.52). Note that $\frac{\mathfrak{m}(K)}{\mathfrak{d}(K)^{2}} \leq c$, for all $K \in \mathcal{T}^{h}$, for all $h$. We end the proof as in lemma using (5.54) instead of (5.53).

In conclusion, recall that in case $1<p \leq 2$, property (iii) of Lemma 1 , which is important for the proof of Lemma 9, holds for families of meshes without any proportionality property (cf. Remark 2). Thus it is natural to ask the following question.

Open problem Is weak proportionality sufficient to have the interpolation property in $W_{0}^{1, p}(\Omega)$ for $1<p \leq 2$ ?

\section{Generalisation to A CASE With CONVECTiON}

The problem

$$
\begin{cases}b(v)_{t}=\operatorname{div} a_{p}(D v-Z(v) \mathbf{e})+f(t, x, v) & \text { on } Q=(0, T) \times \Omega, \\ v=0 & \text { on } \Sigma=(0, T) \times \partial \Omega \\ b(v)(0, \cdot)=u^{0} & \text { on } \Omega\end{cases}
$$

where a given vector $\mathrm{e} \in \mathbb{R}^{d}$ prescribes the direction for convection, can be treated in the same way. In the scalar case, Problem (6.55) appears as a model of certain turbulent flows (e.g., in porous media with nonlinear Darcy law; cf. [12] and their references). Bounded weak solutions of (6.55), for $N=1$, have been studied in [12]. Here, we take assumptions that ensure existence of a weak solution to (6.55) by the Alt-Luckhaus variational approach (cf. [1]), that is

$$
\begin{gathered}
|f(t, x, z)|^{p^{\prime}}+|Z(z)|^{p} \leq C|z|^{\theta p}+M_{1}(t) B(z)^{\theta}+M_{2}(t, x), \\
\text { with some } \theta<1, M_{1} \in L^{1}(0, T) \text { and } M_{2} \in L^{1}(Q) ; \\
Z=\hat{Z} \circ b, \quad \text { where } \hat{Z} \text { is continuous; } \\
f(t, x, \cdot)=\hat{f}(t, x, \cdot) \circ b(\cdot), \quad \text { where } \hat{f} \text { is a Caratheodory function. }
\end{gathered}
$$

The scheme takes the form

$$
\left\{\begin{array}{l}
\mathfrak{m}(K) \frac{b\left(v_{K}^{n}\right)-b\left(v_{K}^{n-1}\right)}{k}=\sum_{L \in \mathcal{N}_{K}} \int_{K L} a_{p}\left(D_{K L L}^{n}(x)-Z_{K L}^{n}(x) \mathbf{e}\right) d x \cdot \nu_{K, L}+\mathfrak{m}(K) f_{K}^{n} \\
\quad \text { for all } K \in \mathcal{T}_{\text {int }}^{h}, n \in\left\{1, \ldots,\left[T / k^{h}\right]+1\right\}, \\
v_{K}^{n}=0 \quad \text { for all } K \in \mathcal{T}_{\text {ext }}^{h}, n \in\left\{1, \ldots,\left[T / k^{h}\right]+1\right\}, \\
u_{K}^{0}=\frac{1}{\mathfrak{m}(K)} \int_{K} u_{0} \quad \text { for all } K \in \mathcal{T}_{\text {int }}^{h} .
\end{array}\right.
$$

Here the values $\left(f_{K}^{n}\right)_{K, n}$ are given by an operator $f^{h}:\left(v_{K}^{n}\right)_{K, n} \mapsto\left(f_{K}^{n}\right)_{K, n}$. We take for instance $f_{K}^{n}=\frac{1}{\left|Q_{K}^{n}\right|} \iint_{Q_{K}^{n}} \hat{f}\left(\tau, y, b\left(v_{K}^{n}\right)\right) d y d \tau$. Further, the values $\left(Z_{K L}^{n}\right)_{K L, n}$ are given by a "discrete convection" operator $\mathcal{Z}^{h}:\left(v_{K}^{n}\right)_{K, n} \mapsto\left(Z_{K L}^{n}(x)\right)_{K L, n}$. We assume that $Z_{K L}^{n}$ belongs to $L^{p}(K L)$ and takes values intermediate between $\hat{Z}\left(b\left(v_{K}^{n}\right)\right)$ and $\hat{Z}\left(b\left(v_{L}^{n}\right)\right)$, i.e., $\max \left\{\left|Z_{K L}^{n}(x)-\hat{Z}\left(b\left(v_{K}^{n}\right)\right)\right|,\left|Z_{K L}^{n}(x)-\hat{Z}\left(b\left(v_{L}^{n}\right)\right)\right|\right\} \leq$ $\left|\hat{Z}\left(b\left(v_{K}^{n}\right)\right)-\hat{Z}\left(b\left(v_{L}^{n}\right)\right)\right|$ for all $x \in K \mid L$. This includes in particular the upwinding choice, usual in numerical implementation (cf. e.g. [17]). 
Under assumptions (6.56),(6.57),(6.58), the solutions of (6.59) converge to a weak solution of (6.55), provided the family of schemes satisfy the admissibility conditions in Theorem 2 . Let us just give the appropriate "continuous" formulation of (6.59).

Set $Z^{h}(t, x)=\sum_{K L, n} Z_{K L}^{n}\left(\mathcal{P}^{h}(x)\right) \mathbb{1}_{I^{n} \times \widehat{K L}}(t, x)$, where $\mathcal{P}^{h}$ is the projection from $K$ to $\partial_{K}$ as defined in Section 2.2. Further, set $f^{h}(t, x)=\sum_{K, n} f_{K}^{n} \mathbb{1}_{Q_{K}^{n}}(t, x)$.

For the sake of simplicity, assume that all the boundary volumes are of zero $d$-dimensional measure. The operator $\mathcal{G}^{h}$ that maps $\eta \in E$ into $\mathcal{G}^{h}[\eta] \in L^{p}(Q)$ defined by

$$
\left\{\begin{array}{l}
\mathcal{G}^{h}[\eta]=\sum_{K, n} D \eta_{K}^{n} \mathbb{1}_{Q_{K}^{n}}, \quad \text { where } \eta_{K}^{n} \in W^{1, p}(K) \text { solves } \\
\operatorname{div} a_{p}(D w-Z(t, x) \mathbf{e})=\frac{1}{\mathfrak{m}(K)} \sum_{L \in \mathcal{N}_{K}} \int_{K L} a_{p}\left(D_{K L}^{n}(x)-Z_{K L}^{n}(x) \mathbf{e}\right) d x \cdot \nu_{K} \quad \text { on } K, \\
\left.a_{p}(D w-Z(t, x) \mathbf{e}) \cdot \nu_{K}\right|_{\partial K}=a_{p}\left(D_{K L}^{n}(x)-Z_{K L}^{n}(x) \mathbf{e}\right) \cdot \nu_{K} \quad \text { for } x \in K L \in \mathcal{E}_{K}, \\
\text { the values of } D_{K L}^{n}(x) \text { are given by }\left(D_{K L}^{n}\right)_{K L, n}=\mathcal{D}^{h}[\eta], \text { and } Z_{K L}^{n}(x)=\left.Z\right|_{I^{n} \times \widehat{K L}}
\end{array}\right.
$$

is the appropriate interpolated gradient operator for Problem (6.55).

Taking $\left(v^{h}\right)_{h}$ a family of interpolated solutions for $\left(\bar{v}^{h}\right)_{h}$, we can write the interpolated formulation of problem (6.59) as

$$
\left\{\begin{array}{l}
u_{t}^{h}=\operatorname{div} a_{p}\left(\mathcal{G}^{h}\left[v^{h}\right]-Z^{h} \mathbf{e}\right)+f^{h} \quad \text { in } E^{\prime} \\
u^{h}(0, \cdot)=u_{0}^{h}(\cdot)
\end{array}\right.
$$

understood in the same sense as in (3.20), (3.21).

Slight changes in the proof of existence of discrete solutions and interpolated gradients, as well as in the $a$ priori estimates and the compactness for the parabolic part are needed. Like in [2, Chap.V], under hypotheses (6.57),(6.58) we can prove that $Z^{h}(t, x) \rightarrow \hat{Z}(b(v(t, x)))$ in $L^{p}(Q)$ and $f^{h}(t, x) \rightarrow \hat{f}(t, x, b(v(t, x)))$ in $L^{p^{\prime}}(Q)$. Adding the convection term leads us to define the consistency as the convergence in $E^{\prime}$ of $\mathcal{A}^{h}[\eta]=-\operatorname{div} a_{p}\left(\mathcal{G}^{h}[\eta]-Z^{h} \mathbf{e}\right)$ to $\mathcal{A}[\eta]=-\operatorname{div} a_{p}(D \eta-Z(v) \mathbf{e})$, for all $\eta \in E$. As in Theorem 3, the idea of the proof of consistency is to approach both $D \eta$ and $Z(v)$ by piecewise constant functions in $L^{p}(Q)$. We obtain in this way the analogs of Theorems 3 and 2 for Problem (6.55).

One could consider more general operators in the elliptic part, of the form $\operatorname{div} \hat{a}(t, x, b(v), D v)$. We need the coercivity, monotonicity and growth assumptions of Leray-Lions-type (cf. $[24,1,2])$ on $\hat{a}$, and some analogs of the inequalities (3.33),(3.34) are required. There is a choice in constructing interpolated gradients, since the dependence of $\hat{a}$ on $t, x$ and $b(v)$ in the analog of (3.24) and (6.60) can be taken into account in various ways. The same proof of consistency can be applied in case $\mathcal{A}^{h}, \mathcal{G}^{h}$ are defined by $\mathcal{A}^{h}[\eta]=-\operatorname{div} a^{h}\left(t, x, \mathcal{G}^{h}[\eta]\right), \mathcal{G}^{h}[\eta]=\sum_{K, n} D \eta_{K}^{n} \mathbb{1}_{Q_{K}^{n}}$, where $\eta_{K}^{n}$ solves

$$
\left\{\begin{array}{l}
\operatorname{div} a^{h}(t, x, D w)=\frac{1}{\mathfrak{m}(K)} \sum_{L \in \mathcal{N}_{K}} \int_{K L} a_{K L}^{n}\left(D_{K L}^{n}(x)\right) d x \cdot \nu_{K, L} \text { on } K \\
\left.a^{h}(t, x, D w) \cdot \nu_{K, L}\right|_{K L}=a_{K L}^{n}\left(D_{K L}^{n}(x)\right) \cdot \nu_{K, L}
\end{array}\right.
$$

with $\left(D_{K L L}^{n}\right)_{K \mid L, n}=\mathcal{D}^{h}[\eta], a^{h}(t, x, \cdot)=\sum_{K \mid L, n} a_{K L}^{n}(\cdot) \mathbb{1}_{I^{n} \times \widehat{K L}}(t, x)$, the functions $\left(a_{K \mid L}^{n}(\cdot)\right)_{K L, n}$ being chosen so that $a^{h}(t, x, \cdot)$ approach $\hat{a}\left(t, x, b\left(\bar{v}^{h}(t, x)\right), \cdot\right)$. 


\section{A Appendix: proofs of AUXiliary ReSUlts}

Proof of Lemma 6: We adapt the proof from [23]. Here, we only need to find a function $\widetilde{\omega}$ on $\mathbb{R}^{+}$ such that

$$
I:=\int_{0}^{T-\tau} \int_{\Omega}\left|u^{h}(t+\Delta t, x)-u^{h}(t, x)\right| d x d t \leq \widetilde{\omega}(\tau) \text { and } \lim _{\tau \rightarrow 0} \widetilde{\omega}(\tau)=0
$$

for all $\Delta t \in[0, \tau]$ and all $h$. The relative compactness of $\left(u^{h}\right)_{h}$ in $L^{1}([0, T] \times \Omega)$ will follow by the Kolmogorov theorem. Note that without loss of generality, we can assume that $\Omega$ is bounded.

Let us construct $\widetilde{\omega}(\cdot)$ verifying (A.61). First fix $h$ and fix $\Delta t \in[0, \tau]$. For $t \in[0, T-\tau]$ set $w^{t}(\cdot)=u^{h}(t+\Delta t, \cdot)-u^{h}(t, \cdot)$. For all $\varphi \in \mathcal{D}(\Omega)$ we have

$$
\int_{\Omega} w^{t}(x) \varphi(x) d x=\int_{t}^{t+\Delta t} \int_{\Omega} \sum_{|\alpha| \leq m} F_{\alpha}^{h}(\theta, x) D^{\alpha} \varphi(x) d x d \theta .
$$

For each $t \in[0, T-\tau]$, take for the test function $\varphi(\cdot)$ a regularisation of $\operatorname{sign} w^{t}(\cdot)$. More exactly, take $\delta>0$, set $\Omega_{-\delta}=\{x \in \Omega: \operatorname{dist}(x, \partial \Omega) \geq \delta\}$ and denote by $\chi_{\Omega_{-\delta}}(\cdot)$ its characteristic function. Choose $\rho \in C_{0}^{\infty}\left([-1,1]^{N}\right), \rho \geq 0$ such that $\int \rho(\sigma) d \sigma=1$ and take in (A.62) $\varphi^{t}(x)=\delta^{-N} \int \rho\left(\frac{x-y}{\delta}\right) \operatorname{sign} w^{t}(y) \chi_{\Omega_{-\delta}}(y) d y$. It is clear that for $|\alpha| \leq m,\left\|D^{\alpha} \varphi^{t}\right\|_{L^{1}([0, T] \times \Omega)} \leq$ const. $\delta^{-m}$ uniformly in $t$ and $h$.

Write $I=I_{1}+I_{2}$, where $I_{1}=\int_{0}^{T-\tau} \int_{\Omega} w^{t}(x) \varphi^{t}(x) d x d t$ and $I_{2}=\int_{0}^{T-\tau} \int_{\Omega}\left(\left|w^{t}(x)\right|-w^{t}(x) \varphi^{t}(x)\right) d x d t$. Using (A.62), we get $\left|I_{1}\right| \leq$ const $\cdot \tau \delta^{-m} \sum_{|\alpha| \leq m}\left\|F_{\alpha}^{h}\right\|_{L^{1}([0, T] \times \Omega)} \leq C_{\Omega} \tau / \delta^{-m}$. By the assumptions of the lemma and the Kolmogorov theorem the family $\left(\int_{0}^{T}\left|u^{h}(t, \cdot)\right| d t\right)_{h}$ is relatively compact in $L^{1}(\Omega)$. Therefore these functions are equiintegrable on $\Omega$, so that $\int_{0}^{T} \int_{\Omega \backslash \Omega_{-2 \delta}}\left|u^{h}(t, x)\right| d x d t \leq \hat{\omega}(\delta)$ with $\lim _{\delta \rightarrow 0} \hat{\omega}(\delta)=0$ uniformly in $h$. Hence

$$
\begin{gathered}
\left|I_{2}\right| \leq 2 \int_{0}^{T-\tau} \int_{K \backslash \Omega_{-2 \delta}}\left|w^{t}(x)\right| d x d t+\int_{0}^{T-\tau} \int_{\Omega_{-2 \delta}}|| w^{t}(x)\left|-w^{t}(x) \int \delta^{-N} \rho\left(\frac{x-y}{\delta}\right) \operatorname{sign} w^{t}(y)\right| d y d x d t \leq \\
\leq 4 \hat{\omega}(\delta)+\int_{0}^{T-\tau} \int_{\Omega_{-2 \delta}} \int \delta^{-N} \rho\left(\frac{x-y}{\delta}\right)|| w^{t}(x)\left|-w^{t}(x) \operatorname{sign} w^{t}(y)\right| d y d x d t .
\end{gathered}
$$

Since for all $a, b \in \mathbb{R}$ we have ||$a|-a \operatorname{sign} b| \leq 2|a-b|$, it follows that

$$
\begin{aligned}
\left|I_{2}\right| & \leq 4 \hat{\omega}(\delta)+2 \int_{0}^{T-\tau} \int_{\Omega_{-2 \delta}} \int \delta^{-N} \rho\left(\frac{x-y}{\delta}\right)\left|w^{t}(x)-w^{t}(y)\right| d y d x d t \leq \\
& \leq 4 \hat{\omega}(\delta)+4 \int \rho(\sigma) \int_{0}^{T} \int_{\Omega_{-2 \delta}}\left|u^{h}(t, x)-u^{h}(t, x-\delta \sigma)\right| d x d t d \sigma \leq 4 \hat{\omega}(\delta)+4 \omega(\delta) .
\end{aligned}
$$

The function $\widetilde{\omega}(\tau)=\inf _{\delta>0}\left\{C_{\Omega} \frac{\tau}{\delta^{m}}+4 \hat{\omega}(\delta)+4 \omega(\delta)\right\}$ majorates $I$ and tends to 0 as $\tau \rightarrow 0$, which ends the proof.

Proof of Lemma 7: The proof is standard (cf., e.g., [25]). For all $\eta \in E$ one has

$$
<-\chi, v-\eta>\geq \liminf _{h \rightarrow 0}<\mathcal{A}^{h}\left[v^{h}\right], v^{h}-\eta>\geq \liminf _{h \rightarrow 0}<\mathcal{A}^{h}[\eta], v^{h}-\eta>=<A[\eta], v-\eta>.
$$

Taking $\eta=v+\lambda \zeta$ with $\lambda \in \mathbb{R}, \zeta \in E$ and letting $\lambda$ increase to zero, one gets $\langle\chi+A[v], \zeta>\leq 0$. As $\lambda$ decreases to zero, the inverse inequality follows, so that $\langle\chi+A[v], \zeta\rangle=0$ for all $\zeta \in E$. 
Proof of Lemma T.1: It is a modification of the corresponding proofs in [1] and [2, Chapter V].

First note that

$$
\begin{gathered}
\iint_{Q}\left|u^{h}(t, x+\Delta x)-u^{h}(t, x)\right| d x d t \leq \\
\leq k^{h} \int_{\Omega}\left|u_{0}^{h}(x+\Delta x)-u_{0}^{h}(x)\right| d x+2 \int_{Q}\left|b\left(\bar{v}^{h}(t, x+\Delta x)\right)-b\left(\bar{v}^{h}(t, x)\right)\right| .
\end{gathered}
$$

Since $u_{0}^{h} \rightarrow u_{0}$ in $L^{1}(\Omega)$, the set $\left\{u_{0}^{h}\right\}_{h}$ is compact in $L^{1}(\Omega)$, so that

$$
\sup _{|\Delta x| \leq \Delta} \int_{\Omega}\left|u_{0}^{h}(x+\Delta x)-u_{0}^{h}(x)\right| d x \leq \omega^{0}(\Delta),
$$

with $\omega^{0}: \mathbb{R}^{+} \mapsto \mathbb{R}^{+}$such that $\omega^{0}(\Delta) \rightarrow 0$ as $\Delta \rightarrow 0$, uniformly in $h$. Moreover, note that $b\left(\bar{v}^{h}\right)$ are equiintegrable on $Q$. Indeed, for all set $F \subset Q$, by (4.42) and Proposition 1(ii), one has

$$
\iint_{F}\left|b\left(\bar{v}^{h}\right)\right| \leq \inf _{\delta>0}\left(\delta \iint_{Q} B\left(\bar{v}^{h}\right)+|F| \sup _{|\zeta| \leq 1 / \delta}|b(\zeta)|\right) \leq \inf _{\delta>0}\left(C \delta+|F| \sup _{|\zeta| \leq 1 / \delta}|b(\zeta)|\right)=\omega^{1}(|F|)
$$

with $\omega^{1}: \mathbb{R}^{+} \mapsto \mathbb{R}^{+}$such that $\omega^{1}(|F|) \rightarrow 0$ as $|F| \rightarrow 0$.

For $M>0$ let us introduce $R_{M}^{h}=\left\{(t, x) \in Q\right.$ s.t. $\left.\left|\bar{v}^{h}(t, x)\right| \leq M,\left|\bar{v}^{h}(t, x+\Delta x)\right| \leq M\right\}$. It follows by Proposition 1(i) and Lemma 1(i) that $\left\|\bar{v}^{h}\right\|_{L^{1}(Q)} \leq C$ uniformly in $h$. Hence, by the Chebyshev inequality, $\left|Q \backslash R_{M}^{h}\right| \leq C / M$ for all $h$. Let $\omega_{b, M}(\cdot)$ be the modulus of continuity of $b(\cdot)$ on $[-M, M]^{N}$. Integrating separately over $Q \backslash R_{M}^{h}$ and $R_{M}^{h}$ in the last term in (A.63), we get, for $h<1$,

$$
\begin{gathered}
\iint_{Q}\left|u^{h}(t, x+\Delta x)-u^{h}(t, x)\right| \leq \\
\omega^{0}(|\Delta x|)+4 \omega^{1}\left(\left|Q \backslash R_{M}^{h}\right|\right)+\int_{R_{M}^{h}} \omega_{b, M}\left(\left|\bar{v}^{h}(t, x+\Delta x)-\bar{v}^{h}(t, x)\right|\right) d x d t .
\end{gathered}
$$

It follows by the concavity of $\omega_{b, M}(\cdot)$, by Lemma 1(ii) and Proposition 1(i) that

$$
\begin{aligned}
& \sup _{|\Delta x| \leq \Delta} \iint_{Q}\left|u^{h}(t, x+\Delta x)-u^{h}(t, x)\right| d x d t \leq \\
& \quad \leq \inf _{M>0, \Delta>0}\left\{\omega^{0}(|\Delta|)+4 \omega^{1}(C / M)+|Q| \omega_{b, M}\left(\frac{1}{|Q|} \iint_{Q}\left|\bar{v}^{h}(t, x+\Delta x)-\bar{v}^{h}(t, x)\right| d x d t\right)\right\}= \\
& \quad=\inf _{M>0, \Delta>0}\left\{\omega^{0}(\Delta)+4 \omega^{1}(C / M)+|Q| \omega_{b, M}(C \Delta)\right\}=\omega_{x}(\Delta),
\end{aligned}
$$

and $\omega_{x}(\Delta) \rightarrow 0$ uniformly in $h$ as $\Delta \rightarrow 0$.

Proof of Lemma T.2: We claim that $\bar{v}^{h} \rightarrow v$ in $L^{1}(Q)$ and $b\left(\bar{v}^{h}\right) \rightarrow u$ in $L^{1}(Q)$, and then apply the monotonicity argument of [9].

Since $v^{h} \rightarrow v$ also in $L^{1}(Q)$, the first claim follows from (2.17). Further, by (3.22) we have

$$
\left\|u^{h}-b\left(\bar{v}^{h}\right)\right\|_{L^{1}(Q)} \leq \sum_{K, n} \iint_{Q_{K}^{n}}\left|u^{h}\left(t+k^{h}, x\right)-u^{h}(t, x)\right| d x d t .
$$

By the compactness of $\left(u^{h}\right)_{h}$ in $L^{1}(Q)$, the right-hand side of (A.64) vanishes as $h \rightarrow 0$.

Without loss of generality, we can assume that $b\left(\bar{v}^{h}\right) \rightarrow u$ a.e. on $Q$. For each $\varepsilon>0$, choose a set $R_{\varepsilon} \subset Q$, with $\left|R_{\varepsilon}\right|<\varepsilon$, such that $v \in L^{\infty}\left(Q \backslash R_{\varepsilon}\right)$ and $b\left(\bar{v}^{h}\right) \rightarrow u$ in $L^{\infty}\left(Q \backslash R_{\varepsilon}\right)$. This is always 
possible, by the Chebyshev inequality and the Egorov Theorem. Consequently, we have $b(v+\lambda \zeta) \rightarrow b(v)$ in $L^{\infty}\left(Q \backslash R_{\varepsilon}\right)$ as $\lambda \in \mathbb{R}$ tends to zero, for all $\zeta \in L^{\infty}(Q)$. Then for all $\eta \in L^{\infty}(Q)$, using the monotonicity of $b(\cdot)$, we get

$$
\begin{aligned}
\iint_{Q \backslash R_{\varepsilon}} u(v-\eta)=\lim _{h \rightarrow 0} \iint_{Q \backslash R_{\varepsilon}} b\left(\bar{v}^{h}\right)\left(\bar{v}^{h}-\eta\right) \geq \\
\quad \geq \lim _{h \rightarrow 0} \iint_{Q \backslash R_{\varepsilon}} b(\eta)\left(\bar{v}^{h}-\eta\right)=\iint_{Q \backslash R_{\varepsilon}} b(\eta)(v-\eta) .
\end{aligned}
$$

Now it is sufficient to take $\eta=v+\lambda \zeta$ with $\lambda \uparrow 0$ and $\lambda \downarrow 0$ in order to deduce that $\pm \int_{Q \backslash R_{\varepsilon}}(u-b(v)) \cdot \zeta \geq 0$. Since $\zeta \in L^{\infty}(Q)$ and $\varepsilon>0$ are arbitrary, $u=b(v)$ a.e. on $Q$.

\section{References}

[1] H.W. Alt And S. Luckhaus, "Quasilinear elliptic-parabolic differential equations", Math. Z. 183 (1983), no. 3, pp 311-341.

[2] B. AndReianov, "Quelques problèmes de la théorie de systèmes paraboliques dégénérés nonlinéares et de lois de conservation", Ph.D. thesis, Laboratoire de Mathématiques, Univ. de Franche-Comté, Besançon, France, 2000.

[3] B. Andreianov, M. Gutnic and P. Wittbold, "L'approche "continue" pour une méthode de volumes finis", C. R. Acad. Sci. Paris Sér. I Math. 332 (2001), no. 5, pp 477-482.

[4] J.W. BARRETt AND W.B. LiU, "Finite element approximation of the parabolic p-Laplacian", SIAM J. Numer. Anal. 31 (1994), no. 2, pp 413-428.

[5] J. BeAR, "Dynamics of Fluids in Porous Media", Elsevier, New-York, 1972.

[6] Рh. BÉnilan And P. Wittbold, "On mild and weak solutions of elliptic-parabolic problems", Adv. Differential Equations 1 (1996), no. 6, pp 1053-1073.

[7] F. Bouhsiss, "Etude d'un problème parabolique par les semi-groupes non linéaires", in Anal. non-linéaire, Publ. Math. UFR Sci. Tech Besançon 15 (1995/97), Univ. de Franche-Comté, Besançon, France, pp 133-141.

[8] H. BRÉZIS, “Analyse fonctionnelle. Théorie et applications", Masson, Paris, 1983.

[9] H. BrÉzis ANd W.A. Strauss, "Semi-linear second-order elliptic equations in $L^{1}$ ”, J. Math. Soc. Japan 25 (1973), pp 565-590.

[10] J. CarRillo AND P. Wittbold, "Uniqueness of renormalized solutions of degenerate elliptic-parabolic problems”, J. Differential Equations 156 (1999), no. 1, pp 93-121.

[11] S.-S. CHOW, "Finite element error estimates for nonlinear elliptic equations of monotone type", Numer. Math. 54 (1989), no. 4, pp 373-393.

[12] J.I. DiAZ AND F. DE ThÉLIN, "On a nonlinear parabolic problem arising in some models related to turbulent flows", SIAM J. Math. Anal. 25 (1994), no. 4, pp 1085-1111.

[13] Yu.V. Egorov and V.A. Kondratiev, "On Spectral Theory of Elliptic Operators", Oper. Theory Adv. and Appli. 89, Birkhäuser Verlag, Basel, 1996. 
[14] R. Eymard, T. Gallouet, M. Gutnic, R. Herbin and D. Hilhorst, "Approximation by the finite volume method of an elliptic-parabolic equation arising in environmental studies", Math. Models Methods Appl. Sci. 11 (2001), no. 9, pp 1505-1528.

[15] R. Eymard, T. Gallouët and R. Herbin, "Finite Volumes Methods", in Handbook of Numerical Analysis, P.G. Ciarlet, J.-L. Lions, eds., North Holland, Amsterdam, 2000, pp 715-1022.

[16] R. Eymard, T. Gallouët, D. Hilhorst and Y. NaÏt Slimane, "Finite volumes and nonlinear diffusion equations", RAIRO Math. Model. Numer. Anal. 32 (1998), no. 6, pp 747-761.

[17] R. Eymard, M. Gutnic And D. Hilhorst, "The finite volume method for an ellipticparabolic equation", Acta Math. Univ. Comenian. (N.S.) 67 (1998), no. 1, pp 181-195.

[18] R. Eymard, M. Gutnic And D. Hilhorst, "The finite volume method for Richards equation", Comput. Geosci. 3 (1999), no. 3-4, pp 259-294 (2000).

[19] R. Glowinski And A. Marrocco, "Sur l'approximation par éléments finis d'ordre un, et la résolution, par pénalisation-dualité, d'une classe de problèmes de Dirichlet non linéaires", RAIRO Anal. Numér. 9 (1975), no. R-2, pp 41-76.

[20] N. JU, "Numerical analysis of the parabolic p-Laplacian: approximation of trajectories", SIAM J. Numer. Anal. 37 (2000), no. 6, pp 1861-1884.

[21] J. KAČUR, "On a solution of degenerate elliptic-parabolic problems in Orlicz-Sobolev spaces I.", Math. Z. 203 (1990), no. 1, pp 153-171.

[22] J. KAČUR, "On a solution of degenerate elliptic-parabolic problems in Orlicz-Sobolev spaces II.", Math. Z. 203 (1990), no. 4, pp 569-579.

[23] S.N. KRUZHKoV, "Results on the nature of the continuity of solutions of parabolic equations and some of their applications", Mat. Zametki 6 (1969), no. 1, pp 97-108 (in Russian); English translation in Math. Notes 6 (1969), no. 1, pp 517-523.

[24] J. LERAY AND J.-L. Lions, "Quelques résultats de Višik sur les problèmes elliptiques non linéaires par les méthodes de Minty-Browder”, Bull. Soc. Math. France 93 (1965), pp 97-107.

[25] J.-L. Lions, "Quelques méthodes de résolution des problèmes aux limites non linéaires", Dunod, Gauthier-Villars, Paris, 1969.

[26] F. Отто, " $L^{1}$-Contraction and uniqueness for quasilinear elliptic-parabolic problems", J. Differential Equations 131 (1996), no. 1, pp 20-38. 